\title{
Light-front representation of chiral dynamics in peripheral transverse densities
}

\author{
C. Granados ${ }^{a}$ and C. Weiss ${ }^{b}$ \\ ${ }^{a}$ Department of Physics and Astronomy, Uppsala University, \\ Box 516, 75120 Uppsala, Sweden \\ ${ }^{b}$ Theory Center, Jefferson Lab, \\ Newport News, VA 23606, U.S.A. \\ E-mail: carlos.granados@physics.uu.se, weiss@jlab.org
}

ABSTRACT: The nucleon's electromagnetic form factors are expressed in terms of the transverse densities of charge and magnetization at fixed light-front time. At peripheral transverse distances $b=O\left(M_{\pi}^{-1}\right)$ the densities are governed by chiral dynamics and can be calculated model-independently using chiral effective field theory (EFT). We represent the leading-order chiral EFT results for the peripheral transverse densities as overlap integrals of chiral light-front wave functions, describing the transition of the initial nucleon to soft pion-nucleon intermediate states and back. The new representation (a) explains the parametric order of the peripheral transverse densities; (b) establishes an inequality between the spin-independent and -dependent densities; (c) exposes the role of pion orbital angular momentum in chiral dynamics; (d) reveals a large left-right asymmetry of the current in a transversely polarized nucleon and suggests a simple interpretation. The light-front representation enables a first-quantized, quantum-mechanical view of chiral dynamics that is fully relativistic and exactly equivalent to the second-quantized, field-theoretical formulation. It relates the charge and magnetization densities measured in low-energy elastic scattering to the generalized parton distributions probed in peripheral high-energy scattering processes. The method can be applied to nucleon form factors of other operators, e.g. the energy-momentum tensor.

KEYwords: Electromagnetic Processes and Properties, Chiral Lagrangians, Parton Model ARXiv EPrint: 1503.04839 


\section{Contents}

1 Introduction 1

2 Transverse densities 4

3 Chiral dynamics in current matrix element $\quad 6$

$\begin{array}{ll}3.1 & \text { Peripheral chiral processes } \\ & \end{array}$

$\begin{array}{lll}3.2 & \text { Overlap representation } & 9\end{array}$

4 Chiral light-front wave function $\quad 12$

$\begin{array}{lll}4.1 & \text { Nucleon spin states } & 12\end{array}$

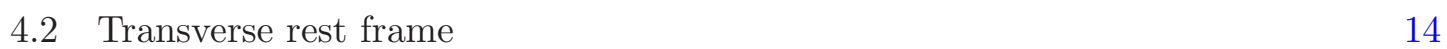

$\begin{array}{ll}4.3 \text { Coordinate representation } & 14\end{array}$

5 Peripheral transverse densities $\quad 16$

$\begin{array}{ll}5.1 & \text { Overlap representation } \\ 5.2 & \text { Chiral }\end{array}$

$\begin{array}{ll}5.2 \text { Chiral order and inequality } & 19\end{array}$

$\begin{array}{ll}5.3 \text { Numerical evaluation } & 19\end{array}$

$\begin{array}{lll}5.4 & \text { Transverse polarization } & 20\end{array}$

$\begin{array}{ll}5.5 & \text { Quantum-mechanical picture } 25\end{array}$

$\begin{array}{ll}5.6 & \text { Contact term } 26\end{array}$

$\begin{array}{ll}5.7 & \text { Region of applicability } 28\end{array}$

6 Chiral generalized parton distributions $\quad 30$

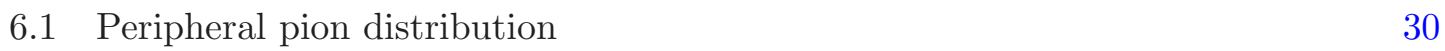

6.2 Charge density from peripheral partons 32

$\begin{array}{lll}7 & \text { Summary and outlook } & 33\end{array}$

A Light-front time-ordered formulation $\quad 35$

\section{Introduction}

Transverse densities have become an essential tool in the analysis of current matrix elements (vector, axial) and the description of the spatial structure of hadrons [1-4]. They are defined as the two-dimensional Fourier transforms of the invariant form factors and describe the distribution of charge and current in the hadron in transverse space at fixed light-front time $x^{+} \equiv t+z$. They are frame-independent (boost-invariant) and provide an objective spatial representation of the hadron as a relativistic system. In the context of 
QCD the transverse densities correspond to a projection of the generalized parton distributions (GPDs) describing the transverse spatial distribution of quarks and antiquarks $[2,5]$; as such they connect the information gained from low-energy elastic scattering with the partonic content probed by high-momentum-transfer processes in high-energy scattering. In composite models of nucleon structure the transverse densities can be expressed as proper densities of the light-front wave functions of the system and are therefore a natural ground for phenomenological analysis. Considerable efforts have been devoted to extracting the transverse charge and magnetization densities in the nucleon from the available electromagnetic (Dirac, Pauli) form factor data and studying their properties $[3,6,7]$; see ref. [4] for a review.

Of particular interest are the densities in the nucleon's chiral periphery. At transverse distances $b=O\left(M_{\pi}^{-1}\right)$, where the pion mass is regarded as parametrically small compared to the typical inverse hadronic size, the densities are governed by the universal dynamics resulting from the spontaneous breaking of chiral symmetry and can be computed from first principles using the methods of chiral effective field theory (EFT). The isovector charge and magnetization densities, $\rho_{1}^{V}(b)$ and $\rho_{2}^{V}(b)$, arise from chiral processes in which the current couples to the nucleon through exchange of a two-pion system with momenta $O\left(M_{\pi}\right)$ relative to the nucleon. A detailed investigation of the properties of the peripheral transverse densities in leading-order (LO) relativistic chiral EFT was performed in ref. [8]. The densities decay exponentially with a range given by the mass of the exchanged system, $2 M_{\pi}$ ("Yukawa tail"); their overall strength and the underlying power-like behavior in $b$ are determined by the coupling of the two-pion exchange to the nucleon and exhibit a rich structure. It was found that the transverse charge density $\rho_{1}^{V}(b)$ and the modified magnetization density $\widetilde{\rho}_{2}^{V}(b) \equiv(\partial / \partial b) \rho_{2}^{V}(b) /\left(2 M_{N}\right)$ are of the same order in the chiral expansion, obey an approximate inequality $\left|\widetilde{\rho}_{2}^{V}(b)\right|<\rho_{1}^{V}(b)$, and are numerically very close at the distances of interest, $b \gtrsim 1 M_{\pi}^{-1}$. These findings represent model-independent features of the nucleon's chiral periphery and call for a simple explanation.

In ref. [8] the peripheral densities were calculated in a dispersive representation, where they are expressed as integrals of the imaginary parts (or spectral functions) of the invariant form factors along the cut in timelike region at $t>4 M_{\pi}^{2}$. This formulation makes it possible to use the well-known chiral EFT results for the invariant form factors and their spectral functions for the calculation of the transverse densities. While it allows one to derive all properties of interest, it does not provide a mechanical picture of the chiral processes as pions "moving about" the nucleon in space and time. Such a picture could be obtained in a time-ordered representation of chiral EFT, where one works with the concepts of instantaneous configurations, time evolution, and the wave function of the system. Since the transverse densities are defined at fixed light-front time $x^{+}$it is natural to adopt light-front quantization [9-11] and follow the evolution of the relevant chiral processes in light-front time. This representation might explain our earlier findings and provide new insight into the structure of the peripheral transverse densities.

Studying the space-time evolution of chiral dynamics in light-front quantization is interesting also for methodological reasons, unrelated to the specific questions posed by transverse densities. The typical momentum of soft pions in the nucleon rest frame is $k=O\left(M_{\pi}\right)$ 
(the velocity is $v=O(1)$ ), and the typical energy of configurations is $E=O\left(M_{\pi}\right)$. Chiral dynamics thus represents an essentially relativistic system, in which pions "appear" and "disappear" through quantum fluctuations and the number of particles is generally not conserved. In equal-time quantization the particle number observed at an instant changes under Lorentz boosts, so that the wave function is essentially frame-dependent and no meaningful particle-based description of the theory can be constructed. In light-front quantization the particle number is invariant under boosts, the wave function is frameindependent, and a natural particle-based description is obtained [11]. It represents the only known formulation that permits a consistent first-quantized particle-based description of chiral processes. Such a representation could significantly advance our understanding of chiral dynamics.

In this article we study the nucleon's transverse charge and magnetization densities in the chiral periphery in a first-quantized particle-based representation of chiral dynamics based on light-front quantization. The LO chiral EFT results for the peripheral densities are expressed in time-ordered form, as the result of a transition of the bare nucleon to a virtual pion-nucleon state mediated by the chiral EFT interactions. The densities appear as overlap integrals of the perturbative light-front wave functions describing the $N \rightarrow \pi N$ transition, which are calculable directly from the chiral Lagrangian. The new representation offers new insight into the structure of peripheral densities and reveals several interesting properties. First, it explains in simple terms the parametric order of the peripheral charge and modified magnetization densities, $\rho_{1}^{V}(b)$ and $\widetilde{\rho}_{2}^{V}(b)$, in the chiral expansion. Second, it proves the inequality $\left|\widetilde{\rho}_{2}^{V}(b)\right|<\rho_{1}^{V}(b)$, which had been observed numerically in the earlier study using the invariant formulation [8]. It also explains why the inequality is approximately saturated, $\left|\widetilde{\rho}_{2}^{V}(b)\right| \approx \rho_{1}^{V}(b)$, and shows that this is related to the essentially relativistic character of chiral dynamics. Third, the wave function overlap representation exposes the role of the pion's orbital angular momentum in the peripheral transverse densities. A particularly simple picture is obtained with transversely polarized nucleon states, where only a single pion orbital with $L=1$ accounts for both densities, and the relation between $\widetilde{\rho}_{2}^{V}(b)$ and $\rho_{1}^{V}(b)$ is explained by the "left-right" asymmetry induced by the orbital motion of the pion in the preferred longitudinal direction. We emphasize that the first-quantized representation developed in the present work is equivalent to the invariant LO chiral EFT expressions used in earlier studies, and that the new insights derived from it reflect general properties of the nucleon's chiral periphery. A summary of our results has been presented in ref. [12].

In the present work we derive the light-front representation of peripheral chiral processes by rewriting the result obtained in Lorentz-invariant chiral EFT. This approach has the advantage that it guarantees equivalence to the well-tested invariant formulation and avoids the use of light-front specific techniques. The $\pi N$ light-front wave function of the nucleon in chiral EFT is defined in terms of the vertex function provided by the chiral Lagrangian, and the wave function overlap representation of the current matrix element is obtained naturally from the reduction of the Feynman integrals. Similar techniques have been used in calculations of elastic form factors in relativistic bound state models of nuclei and hadrons; see refs. [13-16] and references therein. The connection with the conventional light-front time-ordered Hamiltonian approach [11] is explained in appendix A. Some for- 
mal aspects of chiral EFT in the time-ordered formulation were studied in refs. [17-19]. Our derivation also confirms the presence of an instantaneous term (or zero mode contribution) in the light-front representation of the chiral component of the transverse charge density $[18,20]$. This term has a simple physical interpretation as describing the contribution of large-mass (non-chiral) intermediate states in time-ordered chiral processes and is shown to be numerically small.

The light-front representation of chiral dynamics described here can be applied also to the peripheral transverse densities of other local operators, e.g. the matter and momentum densities associated with the energy-momentum tensor, and to the peripheral GPDs probed in high-energy scattering processes. We derive the wave function overlap representation of the light-front "plus" momentum densities of peripheral pions in the nucleon, which determine the chiral component of the nucleon's parton densities at transverse distances $b=O\left(M_{\pi}^{-1}\right)$. This establishes a formal connection between our chiral EFT results for the peripheral transverse densities and the nucleon's quark/antiquark content. The lightfront momentum density of peripheral pions could in principle also be probed directly in peripheral high-energy scattering processes.

The plan of this article is as follows. In section 3 we review the transverse density representation of the current matrix element, the peripheral chiral contributions in the invariant formulation [8], and derive the light-front overlap representation of the current matrix element. In section 4 we investigate the properties of the peripheral $\pi N$ light-front wave function, including the choice of nucleon spin states and the coordinate representation. In section 5 we express the transverse densities $\rho_{1}^{V}$ and $\widetilde{\rho}_{2}^{V}$ as overlap integrals of the coordinate-space $\pi N$ light-front wave functions and study their properties. Using longitudinal nucleon spin states we discuss the parametric order of the densities, derive the inequality between them, and evaluate them numerically. We also present the expressions for transverse nucleon spin states and show that they correspond to a simple mechanical picture of a pion with $L=1$ orbiting around the nucleon in the rest frame. This picture concisely summarizes the dynamical content of the LO chiral EFT contribution and represents the main result of this work. We also compute the instantaneous (contact term) contribution to the densities and show that it is numerically small. In section 6 we connect the transverse densities with the peripheral parton content of the nucleon in QCD. We derive the wave function overlap representation of the pion plus momentum distribution ("pion GPD") in chiral EFT, which determines the nucleon's peripheral parton densities [21, 22], and show that the transverse charge density is recovered by integrating the peripheral quark/antiquark densities over the parton momentum fraction $x$.

\section{Transverse densities}

The transition matrix element of the electromagnetic current between nucleon states is parametrized in terms of two invariant form factors (we follow the notation and conventions of ref. [8])

$$
\left\langle N\left(p_{2}, \sigma_{2}\right)\left|J^{\mu}(x)\right| N\left(p_{1}, \sigma_{1}\right)\right\rangle=\bar{u}_{2}\left[\gamma^{\mu} F_{1}(t)-\frac{\sigma^{\mu \nu} \Delta_{\nu}}{2 M_{N}} F_{2}(t)\right] u_{1} e^{i \Delta x},
$$


where $p_{1,2}$ are the nucleon 4-momenta, $\sigma_{1,2}$ the spin quantum numbers, $u_{1} \equiv u\left(p_{1}, \sigma_{1}\right)$ etc. the nucleon bispinors, normalized to $\bar{u}_{1} u_{1}=\bar{u}_{2} u_{2}=2 M_{N}$, and $\sigma^{\mu \nu} \equiv \frac{1}{2}\left[\gamma^{\mu}, \gamma^{\nu}\right]$. The 4-momentum transfer is defined as

$$
\Delta \equiv p_{2}-p_{1}
$$

and the dependence of the matrix element on the space-time point $x$ where the current is measured is dictated by translational invariance. The Lorentz-invariant momentum transfer is

$$
t \equiv \Delta^{2}=\left(p_{2}-p_{1}\right)^{2}
$$

with $t<0$ in the physical region for electromagnetic scattering. The Dirac and Pauli form factors, $F_{1}$ and $F_{2}$, are invariant functions of $t$ and can be discussed independently of any reference frame.

In the context of the light-front description of nucleon structure one naturally considers the form factors in a frame where the momentum transfer vector lies in the transverse $(x-y)$ plane,

$$
\Delta^{\mu} \equiv\left(\Delta^{0}, \Delta^{x}, \Delta^{y}, \Delta^{z}\right)=\left(0, \Delta_{T}, 0\right), \quad \Delta_{T}=\left(\Delta^{x}, \Delta^{y}\right), \quad t=-\Delta_{T}^{2},
$$

and represents them as Fourier transforms of certain spatial densities [3, 4]

$$
F_{1,2}\left(t=-\boldsymbol{\Delta}_{T}^{2}\right)=\int d^{2} b e^{i \boldsymbol{\Delta}_{T} \cdot \boldsymbol{b}} \rho_{1,2}(b),
$$

where $\boldsymbol{b} \equiv\left(b^{x}, b^{y}\right)$ is a transverse coordinate variable and $b \equiv|\boldsymbol{b}|$. The formal properties of the transverse densities $\rho_{1,2}(b)$ and their physical interpretation have been discussed extensively in the literature [2,4] and are summarized in ref. [8]. They describe the transverse spatial distribution of the light-front plus component of the current, $\mathrm{J}^{+} \equiv$ $J^{0}+J^{z}$, in the nucleon at fixed light-front time $x^{+}$. Specifically, in a state where the nucleon is localized in transverse space at the origin, and polarized in the $y$-direction, the matrix element of the current $J^{+}$at light-front time $x^{+}=0$ and light-front coordinates $x^{-}=0$ and $\boldsymbol{x}_{T}=\boldsymbol{b}$ is given by

$$
\begin{aligned}
\left\langle J^{+}(\boldsymbol{b})\right\rangle_{\text {localized }} & =(\ldots)\left[\rho_{1}(b)+\left(2 S^{y}\right) \cos \phi \widetilde{\rho}_{2}(b)\right], \\
\widetilde{\rho}_{2}(b) & \equiv \frac{\partial}{\partial b}\left[\frac{\rho_{2}(b)}{2 M_{N}}\right]
\end{aligned}
$$

where $(\ldots)$ hides a trivial factor reflecting the normalization of states (see ref. [8] for details); $\cos \phi \equiv b^{x} / b$ is the cosine of the azimuthal angle, and $S^{y}= \pm 1 / 2$ the spin projection in the $y$-direction in the nucleon rest frame (see figure 1 ). The function $\rho_{1}(b)$ describes the spin-independent part of the current; the function $\cos \phi \widetilde{\rho}_{2}(b)$ describes the spin-dependent part of the current in a transversely polarized nucleon.

The spin-dependent part of the current eq. (2.6) changes sign between negative and positive values of $b^{x}$, or "left" and "right" positions when looking at the nucleon along the negative $z$-direction (down from $z=+\infty$; see figure 1 ). The density $\widetilde{\rho}_{2}(b)$ can thus 

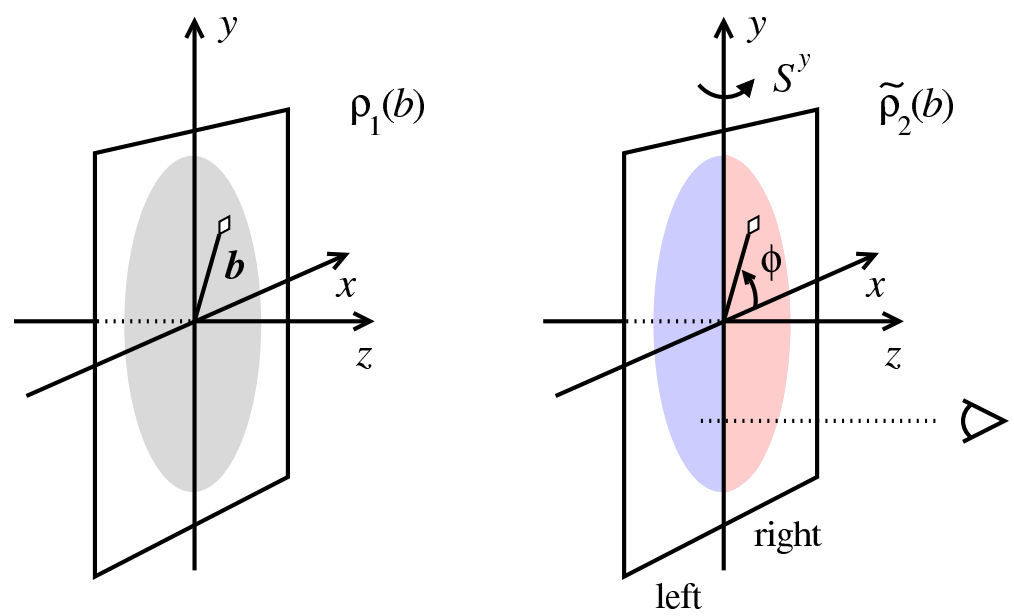

Figure 1. Interpretation of the transverse densities of the electromagnetic current in a nucleon state with spin quantized in the transverse $y$-direction, eq. (2.6). $\rho_{1}(b)$ describes the spin-independent (or left-right symmetric) part of the plus current density; $\cos \phi \widetilde{\rho}_{2}(b)$ describes the spin-dependent (or left-right asymmetric) part.

be interpreted as the left-right asymmetry of the $J^{+}$current in a nucleon polarized in the positive $y$ direction. This interpretation has a natural connection with composite models of nucleon structure, where polarization in the $y$-direction generally induces a convective motion of the constituents around the $y$-axis. As a result, the observer sees the charged constituents on the left side as "blue-shifted" (larger plus momentum), and those on the right ride as "red-shifted" (smaller plus momentum), compared to the unpolarized case [5]. We shall refer to this interpretation in our discussion of the peripheral chiral component in section 5.4. We note that eq. (2.6) and its interpretation can be generalized to the case of arbitrary nucleon polarization states in the rest frame, including non-diagonal transitions; see ref. [8] for details.

The electromagnetic current matrix element and the transverse densities have two isospin components. In the following we are concerned with the isovector component

$$
\langle N|J| N\rangle^{V} \equiv \frac{1}{2}[\langle p|J| p\rangle-\langle n|J| n\rangle], \quad \rho_{1,2}^{V} \equiv \frac{1}{2}\left(\rho_{1,2}^{p}-\rho_{1,2}^{n}\right) .
$$

The isoscalar component is defined by the same expression with the + sign.

\section{Chiral dynamics in current matrix element}

\subsection{Peripheral chiral processes}

At distances $b=O\left(M_{\pi}^{-1}\right)$ the transverse densities are governed by universal chiral dynamics and can be calculated using methods of chiral EFT [8,20]. The isovector charge and magnetization densities in this region arise from chiral processes in which the current couples to the nucleon through exchange of a two-pion system in the $t$-channel. At LO these are the processes described by the Feynman diagrams of figure $2 \mathrm{a}$, where the vertices denote the pion-nucleon couplings of the LO relativistic chiral Lagrangian [23]. They 
produce densities of the form

$$
\rho_{1,2}^{V}(b) \sim P_{1,2}\left(M_{\pi}, M_{N} ; b\right) \exp \left(-2 M_{\pi} b\right)
$$

where the the exponential decay is determined by the minimal mass of the exchanged system, $2 M_{\pi}$; the pre-exponential factors $P_{1,2}$ is determined by the coupling of the exchanged system to the nucleon and exhibits a rich structure due to its dependence on the two scales, $M_{\pi}$ and $M_{N}$. Diagrams in which the current couples directly to the nucleon, or to a pion-nucleon vertex, produce contributions to the densities with range $O\left(M_{N}^{-1}\right)$, or terms $\propto \delta^{(2)}(\boldsymbol{b})$, and do not need to be considered in the calculation of the densities at $b=O\left(M_{\pi}^{-1}\right) \cdot{ }^{1}$ In ref. [8] the densities at $b=O\left(M_{\pi}^{-1}\right)$ resulting from the diagrams of figure $2 \mathrm{a}$ were computed in a dispersive representation, where the densities are expressed as integrals of the imaginary parts of the form factors on the cut at $t>4 M_{\pi}^{2}$, and the integral extends over the parametric region $t-4 M_{\pi}^{2}=O\left(M_{\pi}^{2}\right)$.

Now we want to represent the chiral dynamics generating the peripheral densities as actual processes evolving in light-front time $x^{+}$, and to express the densities in terms of the light-front wave functions of the chiral $\pi N$ system. This could be done by solving the dynamical problem of chiral EFT directly using light-front time-ordered perturbation theory [11]. A more convenient approach is to take the known chiral EFT result in the relativistically invariant formulation and rewrite it such that it corresponds to the overlap of light-front wave functions. This approach maintains the connection with the invariant formulation and naturally generates also the instantaneous terms (zero modes) that require special considerations in the time-ordered approach. In the LO approximation the invariant chiral EFT result for the isovector nucleon current matrix element eq. (2.1) at the position $x=0$ is [24-27] (the specific form here was derived in ref. [8])

$$
\begin{aligned}
\left\langle N_{2}\left|J^{\mu}(0)\right| N_{1}\right\rangle^{V}= & \int \frac{d^{4} k}{(2 \pi)^{4}} \frac{i k^{\mu}}{\left(k_{1}^{2}-M_{\pi}^{2}+i 0\right)\left(k_{2}^{2}-M_{\pi}^{2}+i 0\right)} \\
& \times\left[\frac{g_{A}^{2}}{F_{\pi}^{2}} \frac{\bar{u}_{2} \hat{k}_{2} \gamma_{5}\left(\hat{l}+M_{N}\right) \hat{k}_{1} \gamma_{5} u_{1}}{l^{2}-M_{N}^{2}+i 0}+\frac{1}{F_{\pi}^{2}} \bar{u}_{2} \hat{k} u_{1}\right] \\
& +(\text { diagrams without } \pi \pi \text { cut }),
\end{aligned}
$$

where

$$
k_{1,2}=k \mp \Delta / 2
$$

are the 4-momenta of the pions coupling to the vector current, and the average 4-momentum $k$ was chosen as integration variable. The first term in the brackets results from the triangle diagram in figure $2 \mathrm{a}$ and is proportional to the squared $\pi N N$ coupling $g_{A}^{2} / F_{\pi}^{2}$; it involves the intermediate nucleon propagator with 4-momentum

$$
l \equiv p_{1}-k_{1}=p_{2}-k_{2}
$$

\footnotetext{
${ }^{1}$ The diagrams in which the current couples directly to the nucleon or to a pion-nucleon vertex renormalize the charge in the center of the nucleon, to compensate for the peripheral charge density produced by the two-pion exchange diagrams and ensure overall charge conservation.
} 
(a)

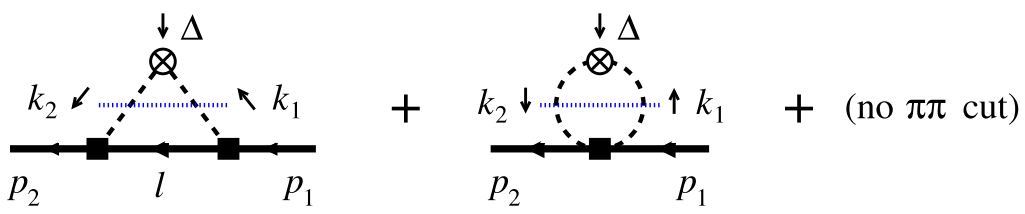

(b)

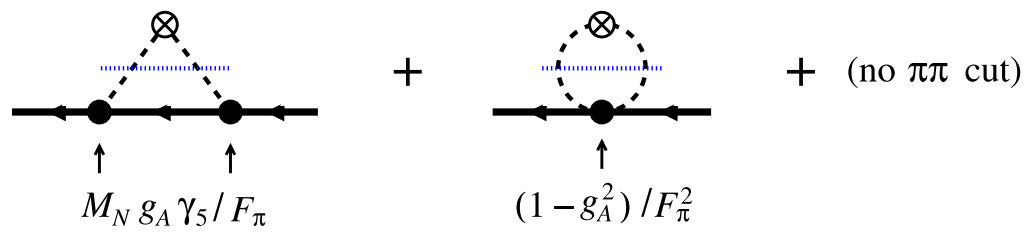

(c)

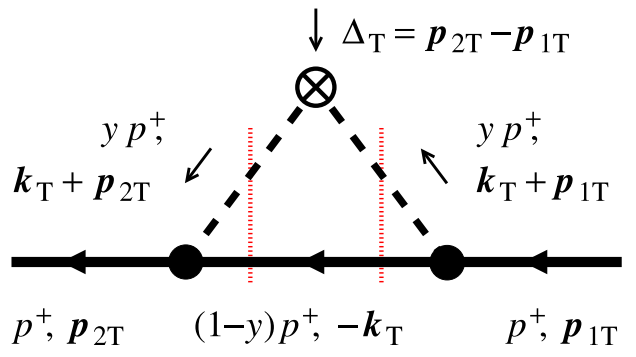

Figure 2. (a) Feynman diagrams of LO chiral EFT processes contributing to the the two-pion cut of the isovector nucleon form factors and the peripheral transverse densities, eq. (3.2). The filled squares denote the vertices of the relativistic chiral Lagrangian (axial vector $\pi N N$ coupling, $\pi \pi N N$ contact coupling). (b) Equivalent representation eqs. (3.7)-(3.9). The filled circles denote the pseudoscalar $\pi N N$ coupling and the effective contact coupling $\propto\left(1-g_{A}^{2}\right)$. (c) Light-front representation of the triangle diagram. The original nucleon makes a transition to a pion-nucleon intermediate state that couples to the current. The plus and transverse momenta are indicated.

(we use the notation $\hat{k}_{1,2} \equiv k_{1,2}^{\mu} \gamma_{\mu}, \hat{l} \equiv l^{\mu} \gamma_{\mu}$ ). The second term results from the contact diagram in figure $2 \mathrm{a}$ and is proportional to the $\pi \pi N N$ contact coupling in the chiral Lagrangian, $1 / F_{\pi}^{2}$. As explained above, eq. (3.2) shows only the contribution from the $\pi \pi$ cut diagrams that contribute to the peripheral density.

The first term in the brackets of eq. (3.2) contains a piece in which the pole of the nucleon propagator cancels, and which is of the same form as the second term. For deriving a wave function overlap representation it is important to extract this piece and combine it with the second term. Expressing the pion momenta as $k_{1,2}=p_{1,2}-l$, cf. eq. (3.4), using the anticommutation relations between the gamma matrices, and the Dirac equation for the external nucleon spinors, $\hat{p}_{1} u_{1}=M_{N} u_{1}$ and $\bar{u}_{2} \hat{p}_{2}=\bar{u}_{2} M_{N}$, we rewrite the bilinear form in the numerator of the first term in eq. (3.2) as

$$
\begin{aligned}
\bar{u}_{2} \hat{k}_{2} \gamma_{5}\left(\hat{l}+M_{N}\right) \hat{k}_{1} \gamma_{5} u_{1}= & -4 M_{N}^{2} \bar{u}_{2} \gamma_{5}\left(\hat{l}+M_{N}\right) \gamma_{5} u_{1} \\
& -\left(l^{2}-M_{N}^{2}\right)\left[\bar{u}_{2} \hat{k} u_{1}+(\text { terms even in } k)\right] .
\end{aligned}
$$

In the second term the factor $l^{2}-M_{N}^{2}$ cancels the pole of the nucleon propagator. Moreover, the terms even under $k \rightarrow-k$ in the parentheses integrate to zero because after canceling 
the nucleon pole the remaining integrand in eq. (3.2) is odd under $k \rightarrow-k$. Altogether, we can thus replace the terms in the bracket in eq. (3.2) by

$$
[\ldots]=\left[-\frac{4 M_{N}^{2} g_{A}^{2}}{F_{\pi}^{2}} \frac{\bar{u}_{2} \gamma_{5}\left(\hat{l}+M_{N}\right) \gamma_{5} u_{1}}{l^{2}-M_{N}^{2}+i 0}+\frac{1-g_{A}^{2}}{F_{\pi}^{2}} \bar{u}_{2} \hat{k} u_{1}\right] .
$$

The peripheral chiral EFT contribution to the current matrix element can therefore be represented as the sum of two terms (see figure $2 \mathrm{~b}$ )

$$
\begin{aligned}
\left\langle N_{2}\left|J^{\mu}(0)\right| N_{1}\right\rangle^{V} & =\langle\ldots\rangle_{\text {interm }}^{V}+\langle\ldots\rangle_{\text {contact }}^{V}, \\
\left\langle N_{2}\left|J^{\mu}(0)\right| N_{1}\right\rangle_{\text {interm }}^{V} & \equiv \frac{4 M_{N}^{2} g_{A}^{2}}{F_{\pi}^{2}} \int \frac{d^{4} k}{(2 \pi)^{4}} \frac{(-i) k^{\mu} \bar{u}_{2} \gamma_{5}\left(\hat{l}+M_{N}\right) \gamma_{5} u_{1}}{\left(k_{1}^{2}-M_{\pi}^{2}+i 0\right)\left(k_{2}^{2}-M_{\pi}^{2}+i 0\right)\left(l^{2}-M_{N}^{2}+i 0\right)} \\
\left\langle N_{2}\left|J^{\mu}(0)\right| N_{1}\right\rangle_{\text {contact }}^{V} & \equiv \frac{1-g_{A}^{2}}{F_{\pi}^{2}} \int \frac{d^{4} k}{(2 \pi)^{4}} \frac{i k^{\mu} \bar{u}_{2} \hat{k} u_{1}}{\left(k_{1}^{2}-M_{\pi}^{2}+i 0\right)\left(k_{2}^{2}-M_{\pi}^{2}+i 0\right)} .
\end{aligned}
$$

The first term, eq. (3.8), contains the intermediate nucleon propagator and is identical in form to the pion loop graph with the usual pseudoscalar $\pi N N$ vertex with effective coupling $g_{\pi N N}=M_{N} g_{A} / F_{\pi}$. This will allow us to derive a wave function overlap representation for this term with the pseudoscalar vertex, which is free of the ambiguities of the momentum-dependent axial vector coupling. The second term, eq. (3.9), represents an effective contact term, combining the explicit $\pi \pi N N$ 4-point vertex in the chiral Lagrangian with the "non-propagating" part of the triangle diagram. The appearance of the combination $1-g_{A}^{2}$ indicates that this term expresses internal structure of the nucleon (for a structureless Dirac fermion $g_{A}=1$ ) and that its contribution is numerically small; cf. the discussion in section 5.6. The two terms in the current matrix element thus have distinct physical meaning and will be discussed separately in the following. We emphasize that the decomposition eqs. (3.7)-(3.9) is obtained by identical rewriting of the original Feynman integrals and does not involve additional approximations.

\subsection{Overlap representation}

The intermediate-nucleon term of the current matrix element can be represented as an overlap integral of light-front wave functions. We derive this representation through a suitable three-dimensional reduction of the Feynman integral eq. (3.8). To this end we go to a class of frames where the momentum transfer has only transverse components, $\mathrm{cf}$. eq. (2.4), such that (see figure 2c)

$$
p_{1}^{+}=p_{2}^{+} \equiv p^{+}, \quad \boldsymbol{p}_{2 T}-\boldsymbol{p}_{1 T}=\boldsymbol{\Delta}_{T}, \quad p_{1}^{-}=\frac{M_{N}^{2}+\boldsymbol{p}_{1 T}^{2}}{p^{+}}, \quad p_{2}^{-}=\frac{M_{N}^{2}+\boldsymbol{p}_{2 T}^{2}}{p^{+}} .
$$

The plus component $p^{+}>0$ is a free parameter, whose choice selects a particular frame in a class of frames related by longitudinal boosts. Likewise, the overall transverse momentum remains unspecified; only the difference $\boldsymbol{p}_{2 T}-\boldsymbol{p}_{1 T}$ is required to be equal to the momentum transfer $\boldsymbol{\Delta}_{T}$. We introduce light-front components of the loop momentum $k^{ \pm} \equiv k^{0} \pm k^{z}$ and $\boldsymbol{k}_{T} \equiv\left(k^{x}, k^{y}\right)$,

$$
\int d^{4} k=\frac{1}{2} \int d k^{+} \int d k^{-} \int d^{2} k_{T}
$$


and express $k^{+}$in terms of the boost-invariant pion momentum fraction $y$,

$$
k^{+}=y p^{+} .
$$

The integrand of eq. (3.8) has simple poles in $k^{-}$, at the values determined by the mass shell conditions for the pion and nucleon 4-momenta. The two poles of the pion propagators and the one of the nucleon propagator lie on opposite sides of the real axis if $0<y<1$. The integral over $k^{-}$can thus be taken by closing the contour around the nucleon pole. At the nucleon pole the pion virtualities take the values

$$
\begin{aligned}
& k_{1}^{2}-M_{\pi}^{2}=-\frac{\left(\boldsymbol{k}_{T}+\bar{y} \boldsymbol{p}_{1 T}\right)^{2}}{\bar{y}}-\frac{y^{2} M_{N}^{2}}{\bar{y}}-M_{\pi}^{2}<0, \\
& k_{2}^{2}-M_{\pi}^{2}=-\frac{\left(\boldsymbol{k}_{T}+\bar{y} \boldsymbol{p}_{2 T}\right)^{2}}{\bar{y}}-\frac{y^{2} M_{N}^{2}}{\bar{y}}-M_{\pi}^{2}<0,
\end{aligned}
$$

where

$$
\bar{y} \equiv 1-y .
$$

These virtualities can be related to the invariant mass differences between states in the light-front time-ordered formulation, in which the external nucleon makes a transition to an intermediate $\pi N$ state and back (see figure 2c). The invariant mass difference for the transition from the initial nucleon state with plus momentum $p^{+}$and transverse momentum $\boldsymbol{p}_{1 T}$ to a pion with $y p^{+}$and $\boldsymbol{k}_{T}+\boldsymbol{p}_{1 T}$ and a nucleon with $\bar{y} p^{+}$and $-\boldsymbol{k}_{T}$ is given by

$$
\begin{aligned}
\Delta \mathcal{M}^{2}\left(y, \boldsymbol{k}_{T}, \boldsymbol{p}_{1 T}\right) & \equiv \frac{\left(\boldsymbol{k}_{T}+\boldsymbol{p}_{1 T}\right)^{2}+M_{\pi}^{2}}{y}+\frac{\boldsymbol{k}_{T}^{2}+M_{N}^{2}}{\bar{y}}-M_{N}^{2}-\boldsymbol{p}_{1 T}^{2} \\
& =\frac{\left(\boldsymbol{k}_{T}+\bar{y} \boldsymbol{p}_{1 T}\right)^{2}+M_{\pi}^{2}}{y}+\frac{\left(\boldsymbol{k}_{T}+\bar{y} \boldsymbol{p}_{1 T}\right)^{2}+M_{N}^{2}}{\bar{y}}-M_{N}^{2} .
\end{aligned}
$$

The invariant mass difference for the transition from the final nucleon state with $p^{+}$and $\boldsymbol{p}_{2 T}$ to a pion with $y p^{+}$and $\boldsymbol{k}_{T}+\boldsymbol{p}_{2 T}$ and a nucleon with $\bar{y} p^{+}$and $-\boldsymbol{k}_{T}$ is given by the same expressions with $\boldsymbol{p}_{1 T} \rightarrow \boldsymbol{p}_{2 T}$. It is easy to see that

$$
\begin{aligned}
-\frac{k_{1}^{2}-M_{\pi}^{2}}{y} & =\Delta \mathcal{M}^{2}\left(y, \boldsymbol{k}_{T}, \boldsymbol{p}_{1 T}\right), \\
-\frac{k_{2}^{2}-M_{\pi}^{2}}{y} & =\Delta \mathcal{M}^{2}\left(y, \boldsymbol{k}_{T}, \boldsymbol{p}_{2 T}\right) .
\end{aligned}
$$

Equations (3.18) and (3.19) allow us to interpret the pion propagators in the Feynman integral as invariant mass denominators. (The origin of the expression eq. (3.16) and its connection with the "energy denominator" in light-front time-ordered perturbation theory are explained in appendix A; this information is not needed for the calculations performed here but important for general understanding.)

Further, at the pole of the nucleon propagator the numerator of eq. (3.8) can be factorized. Since at the pole the 4-momentum $l$ is on the mass shell, the matrix $\hat{l}+M_{N}$ coincides with the projector on physical nucleon spin states and can be represented as

$$
\left(M_{N}+\hat{l}\right)_{\mathrm{on}-\mathrm{shell}}=\sum_{\sigma= \pm 1 / 2} u(l, \sigma) \bar{u}(l, \sigma)
$$


where $u(l, \sigma)$ is a set of nucleon 4 -spinors; the choice of polarization states will be specified below. We thus can write the bilinear form in the numerator as (reverting to the full notation $u_{1} \equiv u\left(p_{1}, \sigma_{1}\right)$ and $\left.u_{2} \equiv u\left(p_{2}, \sigma_{2}\right)\right)$

$$
-\left.\bar{u}_{2} \gamma_{5}\left(\hat{l}+M_{N}\right) \gamma_{5} u_{1}\right|_{\text {on-shell }}=\sum_{\sigma= \pm 1 / 2} u\left(p_{2}, \sigma_{2}\right) i \gamma_{5} \bar{u}(l, \sigma) \bar{u}(l, \sigma) i \gamma_{5} u\left(p_{1}, \sigma_{1}\right) .
$$

Here it is understood that the on-shell 4-momentum $l$ is expressed in terms of the remaining integration variables $y$ and $\boldsymbol{k}_{T}$,

$$
l^{+}=\bar{y} p^{+}, \quad l^{-}=\frac{\left|\boldsymbol{k}_{T}\right|^{2}+M_{N}^{2}}{\bar{y} p^{+}}, \quad \boldsymbol{l}_{T}=-\boldsymbol{k}_{T} .
$$

The bilinear forms appearing on the right-hand side of eq. (3.21) can be related to the vertex function for an $N \rightarrow \pi N$ transition with specified on-shell nucleon momenta and spin, and its complex conjugate. Defining the pseudoscalar vertex function for the transition from the initial nucleon state with momentum $p^{+}$and $\boldsymbol{p}_{1 T}$ and spin $\sigma_{1}$ to a nucleon with momentum $\bar{y} p^{+}$and $-\boldsymbol{k}_{T}$ and spin $\sigma$ as

$$
\Gamma\left(y, \boldsymbol{k}_{T}, \boldsymbol{p}_{1 T} ; \sigma, \sigma_{1}\right) \equiv \frac{g_{A} M_{N}}{F_{\pi}} \bar{u}(l, \sigma) i \gamma_{5} u\left(p_{1}, \sigma_{1}\right),
$$

the vertex for the transition to the final state is given by

$$
\frac{g_{A} M_{N}}{F_{\pi}} u\left(p_{2}, \sigma_{2}\right) i \gamma_{5} \bar{u}(l, \sigma)=\frac{g_{A} M_{N}}{F_{\pi}}\left[\bar{u}(l, \sigma) i \gamma_{5} u\left(p_{2}, \sigma_{2}\right)\right]^{*}=\Gamma^{*}\left(y, \boldsymbol{k}_{T}, \boldsymbol{p}_{2 T} ; \sigma, \sigma_{2}\right),
$$

and multiplying eq. (3.21) by the squared coupling constant we obtain

$$
\left.\frac{g_{A}^{2} M_{N}^{2}}{F_{\pi}^{2}} \bar{u}_{2} \gamma_{5}\left(\hat{l}+M_{N}\right) \gamma_{5} u_{1}\right|_{\text {on-shell }}=\sum_{\sigma= \pm 1 / 2} \Gamma^{*}\left(y, \boldsymbol{k}_{T}, \boldsymbol{p}_{2 T} ; \sigma, \sigma_{2}\right) \Gamma\left(y, \boldsymbol{k}_{T}, \boldsymbol{p}_{1 T} ; \sigma, \sigma_{1}\right) .
$$

We now define the light-front wave function of the initial state in the process of figure $2 \mathrm{c}$ as

$$
\Psi\left(y, \boldsymbol{k}_{T}, \boldsymbol{p}_{1 T} ; \sigma, \sigma_{1}\right) \equiv \frac{\Gamma\left(y, \boldsymbol{k}_{T}, \boldsymbol{p}_{1 T} ; \sigma, \sigma_{1}\right)}{\Delta \mathcal{M}^{2}\left(y, \boldsymbol{k}_{T}, \boldsymbol{p}_{1 T}\right)} ;
$$

the wave function for the final state is given by the same expression with $\boldsymbol{p}_{1 T} \rightarrow \boldsymbol{p}_{2 T}$ and $\sigma_{1} \rightarrow \sigma_{2}$ (i.e., it is the same function but evaluated at a different argument). It is then straightforward to compute the integral over $k^{-}$, and the intermediate-nucleon part of the current matrix element eq. (3.8) becomes

$$
\begin{aligned}
\left\langle N_{2}\left|J^{+}(0)\right| N_{1}\right\rangle_{\text {interm }}^{V} & \equiv\left\langle N\left(p^{+}, \boldsymbol{p}_{2 T}, \sigma_{2}\right)\left|J^{+}(0)\right| N\left(p^{+}, \boldsymbol{p}_{1 T}, \sigma_{1}\right)\right\rangle_{\text {interm }}^{V} \\
& =\frac{\left(2 p^{+}\right)}{2 \pi} \int \frac{d y}{y \bar{y}} \int \frac{d^{2} k_{T}}{(2 \pi)^{2}} \sum_{\sigma} \Psi^{*}\left(y, \boldsymbol{k}_{T}, \boldsymbol{p}_{2 T} ; \sigma, \sigma_{2}\right) \Psi\left(y, \boldsymbol{k}_{T}, \boldsymbol{p}_{1 T} ; \sigma, \sigma_{1}\right) .
\end{aligned}
$$

The original Feynman integral is represented as an overlap integral of the light-front wave functions describing the transition from the initial nucleon state $N_{1}$ to a $\pi N$ intermediate state and back to the final nucleon state $N_{2}$. Equation (3.27) will be our starting point for the analysis of the transverse densities. 
The wave function eq. (3.26) is defined in terms of the vertex function obtained from the chiral Lagrangian and the invariant mass difference of the $N \rightarrow \pi N$ transition. Appendix A shows that this object is identical to the traditional light-front wave function, defined as the transition matrix element between the initial nucleon state and the intermediate pionnucleon state in light-front time-ordered perturbation theory. Regarding isospin the wave function eq. (3.26) is normalized such that it describes the transition $p \rightarrow \pi^{0}+p$, for which the coupling is $g_{\pi^{0} p p} \equiv g_{\pi N N}=g_{A} M_{N} / F_{\pi}$. For the transitions $p \rightarrow \pi^{+}+n$ and $n \rightarrow \pi^{-}+p$, which actually contribute to the isovector electromagnetic current matrix element, the couplings follow from isospin invariance and are $g_{\pi^{+} n p}=g_{\pi^{-} p n}=\sqrt{2} g_{\pi N N}$. The isospin factor $\sqrt{2} \times \sqrt{2}=2$ is included in the prefactor of eq. (3.27).

As explained above, we are interested in the current matrix element only in the (unphysical) region of momentum transfers in the vicinity of the two-pion threshold in the $t$-channel, $t-4 M_{\pi}^{2}=O\left(M_{\pi}^{2}\right)$, and consider the wave function representation eq. (3.27) only in this parametric domain. The restriction to this domain will appear naturally when going over to the coordinate representation and considering the region of transverse distances $b=O\left(M_{\pi}^{-1}\right)$.

\section{Chiral light-front wave function}

\subsection{Nucleon spin states}

To proceed with the evaluation of the overlap formula eq. (3.27) we need to specify the nucleon spin states and obtain explicit expressions for the vertex functions eqs. (3.23) and (3.24). Equation (3.27) can be evaluated with any choice of nucleon spin states (external and internal); the resulting expressions and their interpretation depend on the choice, of course. It is natural to choose the nucleon spin states as light-front helicity states [11]. For a nucleon state with light-front momentum $p^{+}$and $\boldsymbol{p}_{T}$ the light-front helicity spinors are obtained by subjecting the Dirac spinors in the rest frame $\left(p^{+}(\mathrm{RF})=M_{N}, \boldsymbol{p}_{T}(\mathrm{RF})=0\right)$ first to a longitudinal boost from $M_{N}$ to $p^{+}$, and then to a transverse boost from 0 to $\boldsymbol{p}_{T}$. The spinors thus obtained transform in a simple manner under longitudinal and transverse boosts. An explicit representation of the light-front helicity spinors is $[10,11]$

$$
u(p, \sigma) \equiv u\left(p^{+}, \boldsymbol{p}_{T}, \sigma\right)=\frac{1}{\sqrt{2 p^{+}}}\left[p^{+} \gamma^{-}+\left(M_{N}-\gamma_{T} \cdot \boldsymbol{p}_{T}\right) \gamma^{+}\right]\left(\begin{array}{c}
\chi(\sigma) \\
0
\end{array}\right)
$$

where $p$ denotes the on-shell 4-momentum vector $\left(p^{2}=M_{N}^{2}\right), \sigma= \pm 1 / 2$, and $\chi(\sigma)$ are rest frame 2 -spinors for polarization in the positive and negative $z$-direction,

$$
\chi(\sigma=1 / 2)=\left(\begin{array}{l}
1 \\
0
\end{array}\right), \quad \chi(\sigma=-1 / 2)=\left(\begin{array}{l}
0 \\
1
\end{array}\right) .
$$




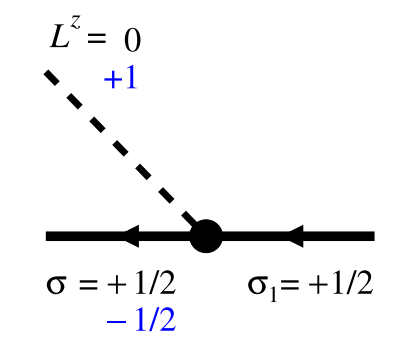

(a)

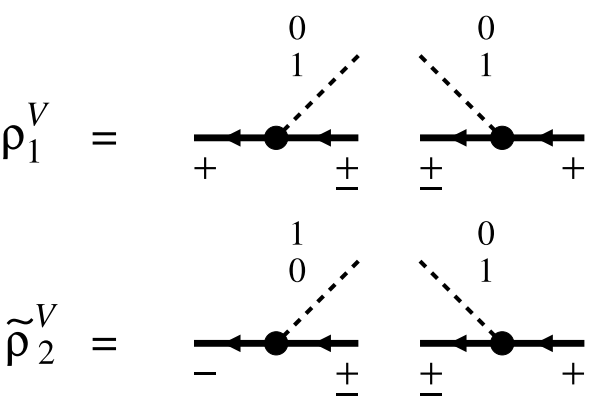

(b)

Figure 3. (a) Spin structure of the $N \rightarrow \pi N$ light-front wave function in chiral EFT. The lightfront helicity corresponds to the $z$-projection of the nucleon spin in the rest frame. The helicityconserving component has orbital angular momentum projection $L^{z}=0$, the helicity-flip component has $L^{z}=1$. (b) Wave function overlap representation of the transverse densities $\rho_{1}^{V}(b)$ and $\widetilde{\rho}_{2}^{V}(b)$ in the light-front helicity representation, eq. (5.4).

The spinors are normalized such that $\bar{u} u=2 M_{N}$ and satisfy the completeness relation eq. (3.20). Using these spinors to evaluate the pseudoscalar vertex eq. (3.23) one gets ${ }^{2}$

$$
\Gamma\left(y, \boldsymbol{k}_{T}, \boldsymbol{p}_{1 T} ; \sigma, \sigma_{1}\right)=\frac{2 i g_{A} M_{N}}{F_{\pi} \sqrt{\bar{y}}}\left[y M_{N} S^{z}\left(\sigma, \sigma_{1}\right)+\left(\boldsymbol{k}_{T}+\bar{y} \boldsymbol{p}_{1 T}\right) \cdot \boldsymbol{S}_{T}\left(\sigma, \sigma_{1}\right)\right],
$$

and similarly for the vertex with $\boldsymbol{p}_{2 T}$ and $\sigma_{2}$. Here $S^{z}$ and $\boldsymbol{S}_{T} \equiv\left(S^{x}, S^{y}\right)$ are the components of the 3 -vector characterizing the spin transition matrix element in the rest frame

$$
S^{i}\left(\sigma, \sigma_{1}\right) \equiv \chi^{\dagger}(\sigma)\left(\frac{1}{2} \sigma^{i}\right) \chi\left(\sigma_{1}\right) \quad(i=x, y, z),
$$

where $\sigma^{i}$ are the Pauli matrices. Use of this compound variable results in a compact representation of the light-front spin structure in close correspondence to non-relativistic quantum mechanics.

The vertex eq. (4.3) contains two structures with different orbital angular momentum (see figure 3). The first term on the right-hand side is diagonal in the light-front helicity, because

$$
S^{z}\left(\sigma, \sigma_{1}\right)=\sigma \delta\left(\sigma, \sigma_{1}\right)
$$

when the rest frame spinors are eigenspinors of $\sigma^{z}$, cf. eq. (4.2). It describes a transition $N \rightarrow \pi N$ in which the nucleon light-front helicity is preserved and the $\pi N$ state has orbital angular momentum projection $L^{z}=0$. The second term is off-diagonal in light-front helicity, because $\sigma^{x}$ and $\sigma^{y}$ have only off-diagonal elements. It corresponds to a transition in which the nucleon helicity is flipped and the $\pi N$ system has orbital angular momentum projection $L^{z}=1$. This is immediately obvious from the fact that this term is proportional to the transverse momentum $\boldsymbol{k}_{T}+\bar{y} \boldsymbol{p}_{1 T}$, which transforms as 2-dimensional vector under rotations around the $z$-axis.

\footnotetext{
${ }^{2}$ The sign of the three-dimensional expressions for the vertex function depends on the convention for the matrix $\gamma^{5}$. We use the Bjorken-Drell convention $\gamma^{5}=i \gamma^{0} \gamma^{1} \gamma^{2} \gamma^{3}$.
} 


\subsection{Transverse rest frame}

Equation (3.27) represents the current matrix element as an overlap integral of the lightfront wave functions of the initial and final nucleon states with overall transverse momenta $\boldsymbol{p}_{1 T}$ and $\boldsymbol{p}_{2 T}$. For further analysis it will be convenient to express these wave functions in terms of the wave function at zero overall transverse momentum (transverse rest frame), such that the overlap integral becomes a quadratic form in a single function. This can be accomplished using the transformation properties under transverse boosts. As can be seen from eqs. (3.26), (3.17) and (4.3), the wave function at overall transverse momentum $\boldsymbol{p}_{1 T}$ is related to that at zero transverse momentum by

$$
\Psi\left(y, \boldsymbol{k}_{T}, \boldsymbol{p}_{1 T} ; \sigma, \sigma_{1}\right)=\Psi\left(y, \boldsymbol{k}_{T}+\bar{y} \boldsymbol{p}_{1 T}, \mathbf{0} ; \sigma, \sigma_{1}\right) \equiv \Psi\left(y, \boldsymbol{k}_{T}+\bar{y} \boldsymbol{p}_{1 T} ; \sigma, \sigma_{1}\right)
$$

The expression for the rest frame wave function can be obtained by setting $\boldsymbol{p}_{1 T}=0$ in eqs. (3.17) and (4.3). For reference we quote the explicit formulas:

$$
\begin{aligned}
\Psi\left(y, \widetilde{\boldsymbol{k}}_{T} ; \sigma, \sigma_{1}\right) & \equiv \frac{\Gamma\left(y, \widetilde{\boldsymbol{k}}_{T} ; \sigma, \sigma_{1}\right)}{\Delta \mathcal{M}^{2}\left(y, \widetilde{\boldsymbol{k}}_{T}\right)}, \\
\Delta \mathcal{M}^{2}\left(y, \widetilde{\boldsymbol{k}}_{T}\right) & \equiv \frac{\widetilde{\boldsymbol{k}}_{T}^{2}+M_{\pi}^{2}}{y}+\frac{\widetilde{\boldsymbol{k}}_{T}^{2}+M_{N}^{2}}{\bar{y}}-M_{N}^{2} \\
\Gamma\left(y, \widetilde{\boldsymbol{k}}_{T} ; \sigma, \sigma_{1}\right) & =\frac{2 i g_{A} M_{N}}{F_{\pi} \sqrt{\bar{y}}}\left[y M_{N} S^{z}\left(\sigma, \sigma_{1}\right)+\widetilde{\boldsymbol{k}}_{T} \cdot \boldsymbol{S}_{T}\left(\sigma, \sigma_{1}\right)\right],
\end{aligned}
$$

where we use $\widetilde{\boldsymbol{k}}_{T}$ to denote the transverse momentum argument. Similar formulas apply to the outgoing wave function with transverse momentum $\boldsymbol{p}_{2 T}$ and spin $\sigma_{2}$. The current matrix element eq. (3.27) can therefore equivalently be expressed in terms of the rest frame wave function,

$$
\begin{aligned}
\left\langle N_{2}\left|J^{+}(0)\right| N_{1}\right\rangle_{\text {interm }}^{V}= & \frac{\left(2 p^{+}\right)}{2 \pi} \int \frac{d y}{y \bar{y}} \int \frac{d^{2} k_{T}}{(2 \pi)^{2}} \\
& \times \sum_{\sigma} \Psi^{*}\left(y, \boldsymbol{k}_{T}+\bar{y} \boldsymbol{p}_{2 T} ; \sigma, \sigma_{2}\right) \Psi\left(y, \boldsymbol{k}_{T}+\bar{y} \boldsymbol{p}_{1 T} ; \sigma, \sigma_{1}\right) .
\end{aligned}
$$

We shall use this expression in our theoretical studies in the following.

\subsection{Coordinate representation}

It is instructive to study the rest frame light-front wave function in the transverse coordinate representation. The coordinate-space wave function allows us to identify the parametric regime of peripheral distances where chiral dynamics is valid and to calculate the transverse densities directly in coordinate space. We define the coordinate-space wave function as the transverse Fourier transform of the momentum-space wave function at fixed plus momentum fraction $y$,

$$
\Phi\left(y, \boldsymbol{r}_{T}, \sigma, \sigma_{1}\right) \equiv \int \frac{d^{2} \widetilde{k}_{T}}{(2 \pi)^{2}} e^{i \boldsymbol{r}_{T} \cdot \widetilde{\boldsymbol{k}}_{T}} \Psi\left(y, \widetilde{\boldsymbol{k}}_{T} ; \sigma, \sigma_{1}\right) .
$$


The vector $\boldsymbol{r}_{T}$ is the difference in the transverse positions of the $\pi$ and $N$ (relative transverse coordinate), so that the wave function describes the physical transverse size distribution of the $\pi N$ system. Because of the spin structure of the vertex eq. (4.7) the coordinatespace wave function can be expressed in terms of two transverse radial wave functions (i.e., scalars with respect to rotations around the $z$-axis),

$$
\Phi\left(y, \boldsymbol{r}_{T}, \sigma, \sigma_{1}\right)=-2 i S^{z}\left(\sigma, \sigma_{1}\right) U_{0}\left(y, r_{T}\right)+\frac{2 \boldsymbol{r}_{T} \cdot \boldsymbol{S}_{T}\left(\sigma, \sigma_{1}\right)}{r_{T}} U_{1}\left(y, r_{T}\right),
$$

where $r_{T} \equiv\left|\boldsymbol{r}_{T}\right|$ is the modulus of the transverse coordinate. Following section 4.1 these are the components with orbital angular momentum projection $L^{z}=0$ and 1 . The Fourier integral is easily calculated by writing the invariant mass in the denominator of the momentumspace wave function, eq. (4.8), in the form

$$
\begin{aligned}
\Delta \mathcal{M}^{2} & =\frac{\widetilde{\boldsymbol{k}}_{T}^{2}+M_{T}^{2}}{y \bar{y}}, \\
M_{T} & \equiv M_{T}(y) \equiv \sqrt{\bar{y} M_{\pi}^{2}+y^{2} M_{N}^{2}},
\end{aligned}
$$

which is the $y$-dependent effective mass governing the transverse momentum dependence. We obtain

$$
\left.\begin{array}{l}
U_{0}\left(y, r_{T}\right) \\
U_{1}\left(y, r_{T}\right)
\end{array}\right\}=\frac{g_{A} M_{N} y \sqrt{\bar{y}}}{2 \pi F_{\pi}}\left\{\begin{array}{r}
y M_{N} K_{0}\left(M_{T} r_{T}\right) \\
M_{T} K_{1}\left(M_{T} r_{T}\right)
\end{array}\right\},
$$

where $K_{0}$ and $K_{1}$ are the modified Bessel functions. At large values of the argument they behave as

$$
K_{0,1}\left(M_{T} r_{T}\right) \sim \sqrt{\frac{\pi}{2}} \frac{e^{-M_{T} r_{T}}}{\sqrt{M_{T} r_{T}}} \quad\left(M_{T} r_{T} \gg 1\right) .
$$

The coordinate-space wave functions fall off exponentially at large transverse distances $r_{T}$, with a width that is given by the transverse mass eq. (4.14) and depends on the pion momentum fraction $y$. This behavior can directly be traced to the singularity of the momentum-space wave function at zero invariant mass, $\Delta \mathcal{M}^{2}=0$, which occurs at complex values of the transverse momentum, $\widetilde{\boldsymbol{k}}_{T}^{2}=-M_{T}^{2}$, cf. eq. (4.13).

The parametric domain in which we consider the coordinate-space wave function is

$$
y=O\left(M_{\pi} / M_{N}\right), \quad r_{T}=O\left(M_{\pi}^{-1}\right) .
$$

In momentum space this corresponds to the region where the pion's light-front momentum components in the nucleon rest frame are

$$
\widetilde{k}^{+}=y M_{N}=O\left(M_{\pi}\right), \quad\left|\widetilde{\boldsymbol{k}}_{T}\right|=O\left(M_{\pi}\right),
$$

and also $\widetilde{k}^{-}=\left(\left|\widetilde{\boldsymbol{k}}_{T}\right|^{2}+M_{\pi}^{2}\right) / \widetilde{k}^{+}=O\left(M_{\pi}\right)$, such that all components of the pion's 4momentum are $O\left(M_{\pi}\right)$ ("soft pion"). In this region chiral dynamics is applicable, and the approximations made in evaluating the peripheral contributions to the current matrix element are self-consistent. Equation (4.14) shows that for momentum fractions $y=O\left(M_{\pi} / M_{N}\right)$

$$
M_{T}(y)=O\left(M_{\pi}\right) \quad\left[y=O\left(M_{\pi} / M_{N}\right)\right]
$$

so that the exponential range of the coordinate-space wave function is indeed of the order $O\left(M_{\pi}^{-1}\right)$, cf. eq. (4.16). 
We note that for $y=O(1)$ the effective mass eq. (4.14) is $M_{T}=O\left(M_{N}\right)$, so that the range of the wave function eq. (4.15) is $O\left(M_{N}^{-1}\right)$. While the wave is still formally defined by eq. (4.15), it does not correspond to a chiral long-distance contribution in this case. This region does not contribute to the peripheral transverse densities, as the wave functions for $y=O(1)$ are exponentially small if the distance is kept at values $r_{T}=O\left(M_{\pi}^{-1}\right)$. In the calculations in section 5 we can thus formally integrate up to $y=1$ without violating the parametric restriction eq. (4.17).

It is interesting to compare the parametric order of the light-front helicity-nonflip $\left(L^{z}=0\right)$ and flip $\left(L^{z}=1\right)$ components of the coordinate-space wave function in $M_{\pi} / M_{N}$. Inspection of eq. (4.15) shows that for $y=O\left(M_{\pi} / M_{N}\right)$ and $r_{T}=O\left(M_{\pi}\right)$, eq. (4.17),

$$
U_{0} / U_{1}=O(1)
$$

The helicity-nonflip and flip components are thus of the same order in the region of interest. ${ }^{3}$ Regarding the numerical values we note that

$$
U_{0}\left(y, r_{T}\right)<U_{1}\left(y, r_{T}\right) \quad\left(0<y<1, r_{T}>0\right),
$$

because $M_{T}(y)>y M_{N}$, cf. eq. (4.14), and $K_{1}(z)>K_{0}(z)$ for all $z>0$. The radial wave functions thus obey a numerical inequality at all values of the argument.

Figure 4 shows a plot of the peripheral radial wave functions $U_{0,1}\left(y, r_{T}\right)$ as functions of the pion momentum fraction $y$. Plot (a) compares $U_{0}$ and $U_{1}$ at a fixed transverse separation. One sees that the $L^{z}=0$ and $L^{z}=1$ components become equal at $y \rightarrow 1$ (i.e., at values several times $M_{\pi} / M_{N}$ ), but show different power-like behavior at $y \rightarrow 0$, as is already apparent from the analytic formulas eq. (4.15). One also sees that the inequality eq. (4.21) is satisfied. Plot (b) shows the $L^{z}=1$ wave function $U_{1}$ at several transverse separations. One sees that values of $y \sim 1$ are strongly suppressed with increasing transverse separation, and that the maximum of the wave function in $y$ shifts to smaller values, in accordance with general expectations. At $r_{T}=$ several times $M_{\pi}^{-1}$ the wave function - and in particular the probabilities - are strongly concentrated at pion momentum fractions $y=O\left(M_{\pi} / M_{N}\right)$, and the parametric approximations are borne out by the numerical results.

\section{$5 \quad$ Peripheral transverse densities}

\subsection{Overlap representation}

We now want to express the peripheral transverse densities in the nucleon in terms of the chiral light-front wave functions. For this we first need to obtain explicit expressions for the invariant form factors in terms of the spin components of the current matrix element eq. (2.1). Taking the nucleon spin states in eq. (2.1) as light-front helicity states,

\footnotetext{
${ }^{3}$ At exceptionally small pion momentum fractions $y \ll M_{\pi} / M_{N}$ the helicity-nonflip component of the wave function vanishes faster than the helicity-flip one, $U_{0} / U_{1} \rightarrow 0$. This scenario is realized in the "molecular" region described in ref. [8].
} 


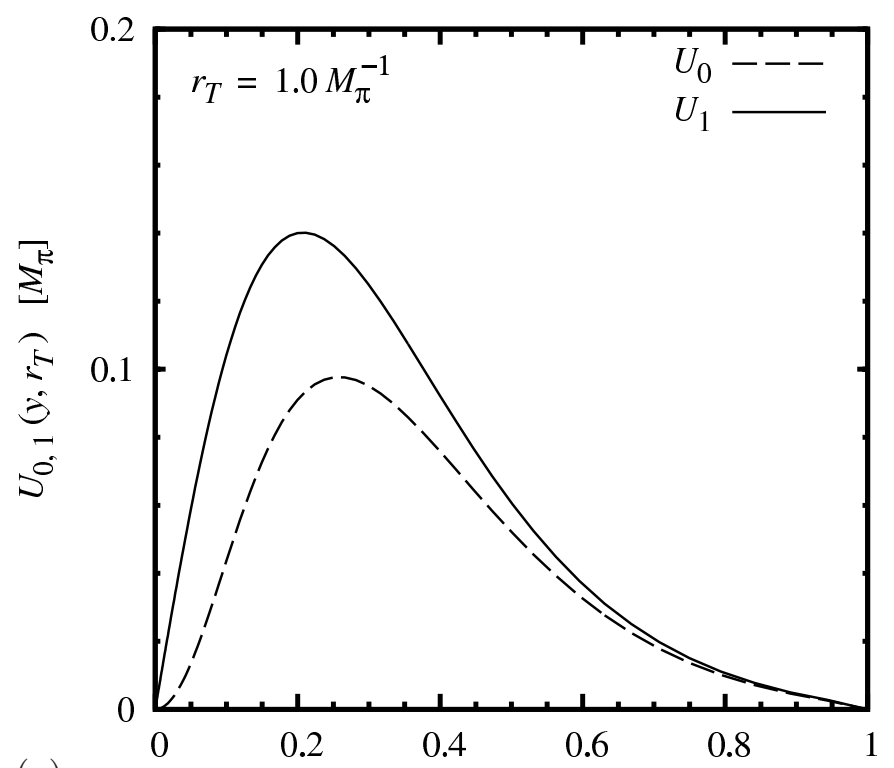

(a)

$y$

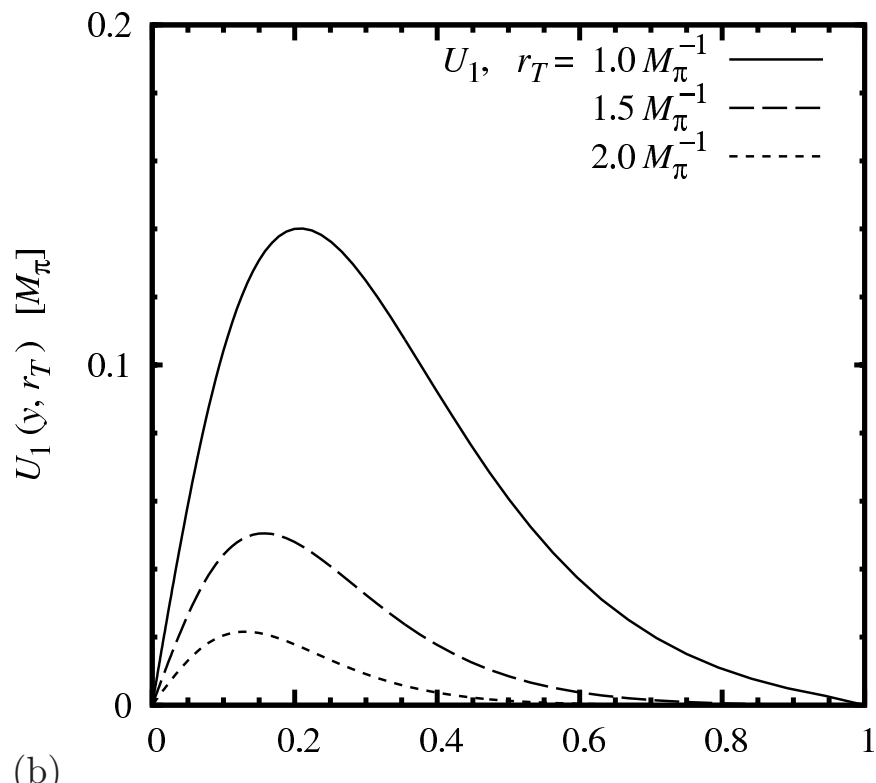

(b)

$y$

Figure 4. Peripheral chiral light-front wave function in coordinate representation. (a) Radial wave functions $U_{0}$ and $U_{1}$, eq. (4.15) as functions of the pion momentum fraction $y$, at fixed transverse separation $r_{T}=1.0 M_{\pi}^{-1}$. (b) Radial wave function $U_{1}$ as function of $y$ at several transverse separations, $r_{T}=(1.0,1.5,2.0) M_{\pi}^{-1}$.

cf. eq. (4.1), and choosing a frame where the momentum transfer has only transverse components, cf. eq. (2.4), the matrix element of the plus component of the current has the form

$$
\begin{aligned}
\left\langle N_{2}\left|J^{+}(0)\right| N_{1}\right\rangle & \equiv\left\langle N\left(p^{+}, \boldsymbol{p}_{2 T}, \sigma_{2}\right)\left|J^{+}(0)\right| N\left(p^{+}, \boldsymbol{p}_{1 T}, \sigma_{1}\right)\right\rangle \\
& =\left(2 p^{+}\right)\left[\delta\left(\sigma_{2}, \sigma_{1}\right) F_{1}\left(-\boldsymbol{\Delta}_{T}^{2}\right)+i\left(\boldsymbol{\Delta}_{T} \times \boldsymbol{e}_{z}\right) \cdot \boldsymbol{S}_{T}\left(\sigma_{2}, \sigma_{1}\right) \frac{F_{2}\left(-\boldsymbol{\Delta}_{T}^{2}\right)}{M_{N}}\right],
\end{aligned}
$$


where $\boldsymbol{S}_{T}$ is defined as in eq. (4.4) in terms of the rest-frame 2 -spinors describing the initial and final nucleon, and $\boldsymbol{e}_{z}$ is the unit vector in $z$-direction. The form factors $F_{1}$ and $F_{2}$ are then obtained from the diagonal and off-diagonal matrix elements as ${ }^{4}$

$$
\left.\begin{array}{c}
F_{1}\left(-\boldsymbol{\Delta}_{T}^{2}\right) \\
F_{2}\left(-\boldsymbol{\Delta}_{T}^{2}\right)
\end{array}\right\}=\frac{1}{2 p^{+}} \sum_{\sigma_{1} \sigma_{2}}\left\langle N_{2}\left|J^{+}(0)\right| N_{1}\right\rangle\left\{\begin{array}{c}
\frac{1}{2} \delta\left(\sigma_{1}, \sigma_{2}\right) \\
\frac{2 M_{N}}{\boldsymbol{\Delta}_{T}^{2}}(-i)\left(\boldsymbol{\Delta}_{T} \times \boldsymbol{e}_{z}\right) \cdot \boldsymbol{S}_{T}\left(\sigma_{1}, \sigma_{2}\right)
\end{array}\right\} .
$$

For the intermediate-nucleon part of the current matrix element we now substitute the overlap representation in terms of the light-front wave functions in the transverse rest frame, eq. (4.10). Using the coordinate representation the overlap integral becomes diagonal in the transverse relative coordinate $\boldsymbol{r}_{T}$ and takes the form

$$
\left\langle N_{2}\left|J^{+}(0)\right| N_{1}\right\rangle_{\text {interm }}^{V}=\frac{\left(2 p^{+}\right)}{2 \pi} \int \frac{d y}{y \bar{y}} \int d^{2} r_{T} e^{-i \bar{y} \boldsymbol{r}_{T} \boldsymbol{\Delta}_{T}} \sum_{\sigma} \Phi^{*}\left(y, \boldsymbol{r}_{T} ; \sigma, \sigma_{2}\right) \Phi\left(y, \boldsymbol{r}_{T} ; \sigma, \sigma_{1}\right) .
$$

Notice that the momentum transfer $\boldsymbol{\Delta}_{T}$ is Fourier-conjugate not to $\boldsymbol{r}_{T}$ itself but to $\bar{y} \boldsymbol{r}_{T}$, which is a general feature of light-front kinematics. It is now straightforward to evaluate the spin sums in eq. (5.2) and obtain the invariant form factors in terms of the $L^{z}=0$ and 1 components of the coordinate-space wave function eq. (4.12). We immediately quote the results for the isovector transverse densities $\rho_{1}^{V}$ and $\widetilde{\rho}_{2}^{V}$, eq. (2.7):

$$
\left.\begin{array}{l}
\rho_{1}^{V}(b) \\
\tilde{\rho}_{2}^{V}(b)
\end{array}\right\}=\frac{1}{2 \pi} \int \frac{d y}{y \bar{y}^{3}}\left\{\begin{array}{c}
{\left[U_{0}(y, b / \bar{y})\right]^{2}+\left[U_{1}(y, b / \bar{y})\right]^{2}} \\
-2 U_{0}(y, b / \bar{y}) U_{1}(y, b / \bar{y})
\end{array}\right\} .
$$

The form of eq. (5.4) is explained by the spin structure of the transitions (see figure $3 \mathrm{~b}$ ). The light-front wave function has a nucleon helicity-conserving $\left(U_{0}\right)$ and a helicity-flipping component $\left(U_{1}\right)$. The current matrix element with the same nucleon helicity in the initial and final state requires the combination of two helicity-conserving or two helicity-flipping wave functions $\left(U_{0}^{2}\right.$ or $\left.U_{1}^{2}\right)$, whereas the matrix element with different nucleon helicities in the initial and final state requires combination of one helicity-conserving and one helicityflipping wave function $\left(U_{0} U_{1}\right)$.

The transverse charge density $\rho_{1}^{V}(b)$ also receives a contribution from the effective contact term in the current matrix element, eq. (3.9). This contribution cannot be represented as an overlap of $\pi N$ light-front wave functions and has to be added to eq. (5.4) as a separate term. The exact form of this term and its interpretation are discussed in section 5.6. The numerical contribution of the contact term turns out to be very small at distances $b=$ few $M_{\pi}^{-1}$, so that the entire $\rho_{1}^{V}$ is to good approximation given by the wave function overlap eq. (5.4). We may therefore compare the properties of the densities $\rho_{1}^{V}$ and $\widetilde{\rho}_{2}^{V}$ on the basis of eq. (5.4) (the contact term is absent in $\widetilde{\rho}_{2}^{V}$ ).

\footnotetext{
${ }^{4}$ The identification of the different spin components can be done conveniently by writing both sides of eq. (5.1) as bilinear forms in the nucleon two-spinors, stripping off the two-spinors, and treating the equation as a $2 \times 2$ matrix equation. The different components can then be projected out by taking appropriate traces of both sides.
} 


\subsection{Chiral order and inequality}

The overlap representation eq. (5.4) reveals several interesting properties of the chiral component of the peripheral transverse densities. First, because the light-front helicityconserving and -flipping wave functions appear in the same order of the chiral expansion (their coefficients involve the same power of $M_{\pi} / M_{N}$ ), cf. eq. (4.20), we conclude that the peripheral densities $\rho_{1}^{V}$ and $\widetilde{\rho}_{2}^{V}$ are of the same order in the chiral expansion,

$$
\widetilde{\rho}_{2}^{V}(b) / \rho_{1}^{V}(b)=O(1) \quad\left[b=O\left(M_{\pi}^{-1}\right)\right] .
$$

While this circumstance was noted earlier in the dispersive approach [8], where it is encoded in the chiral order of the spectral functions of the form factors near threshold, the overlap representation exhibits it more directly and provides a more immediate physical explanation. Notice that the original transverse magnetization density $\rho_{2}^{V}$ is parametrically larger than $\widetilde{\rho}_{2}^{V}$ by a power of $M_{N} / M_{\pi}$, because the spatial derivative in eq. (2.7) "counts" as $O\left(M_{\pi}\right)$ in the region $b=O\left(M_{\pi}^{-1}\right)$, and thus

$$
\rho_{2}^{V}(b) / \rho_{1}^{V}(b)=O\left(M_{N} / M_{\pi}\right) .
$$

The parametric equality of $\rho_{1}^{V}$ and $\widetilde{\rho}_{2}^{V}$, eq. (5.5), allows for non-trivial dynamical relations between the two densities and provides additional motivation for working with $\widetilde{\rho}_{2}^{V}$ rather than $\rho_{2}^{V}$.

Second, we observe an inequality between the peripheral transverse densities,

$$
\left|\widetilde{\rho}_{2}^{V}(b)\right| \leq\left|\rho_{1}^{V}(b)\right|
$$

It follows from the inequality obeyed by the quadratic forms in the integrands of the $y$ integral in eq. (5.4), $U_{0}^{2}+U_{1}^{2} \geq 2 U_{0} U_{1}$. While eq. (5.7) was observed accidentally in the numerical calculations of ref. [8], its mathematical proof and physical explanation become possible with the wave function overlap representation provided here. Recalling that $\rho_{1}^{V}(b)$ and $\widetilde{\rho}_{2}^{V}(b)$ represent the spin-independent and -dependent components of the "plus" current at transverse position $b$ in a nucleon localized at the origin and polarized along the $y$-direction, cf. eq. (2.6) and figure 1, we see that the inequality eq. (5.7) implies the positivity condition

$$
\left\langle J^{+}(\boldsymbol{b})\right\rangle_{\text {localized }} \geq\left|\rho_{1}^{V}(b)\right|-\left|\tilde{\rho}_{2}^{V}(b)\right| \geq 0 .
$$

This property appears natural when one realizes that the plus current at $b=O\left(M_{\pi}^{-1}\right)$ results from peripheral pions, and that the current carried by an on-shell pion is proportional to its 4-momentum, $\left\langle\pi^{+}(k)\left|J^{+}\right| \pi^{+}(k)\right\rangle=2 k^{+}>0$. Such a "quantum-mechanical" picture of the peripheral densities will be explored further in section 5.5 .

\subsection{Numerical evaluation}

We now want to use the overlap representation eq. (5.4) to study the numerical behavior of the transverse densities. Figure 5 shows the integrands of $\rho_{1}(b)$ and $-\widetilde{\rho}_{2}(b)$ as functions of the pion momentum fraction $y$ at a fixed transverse distance $b=1.0 M_{\pi}^{-1}$. One sees that 


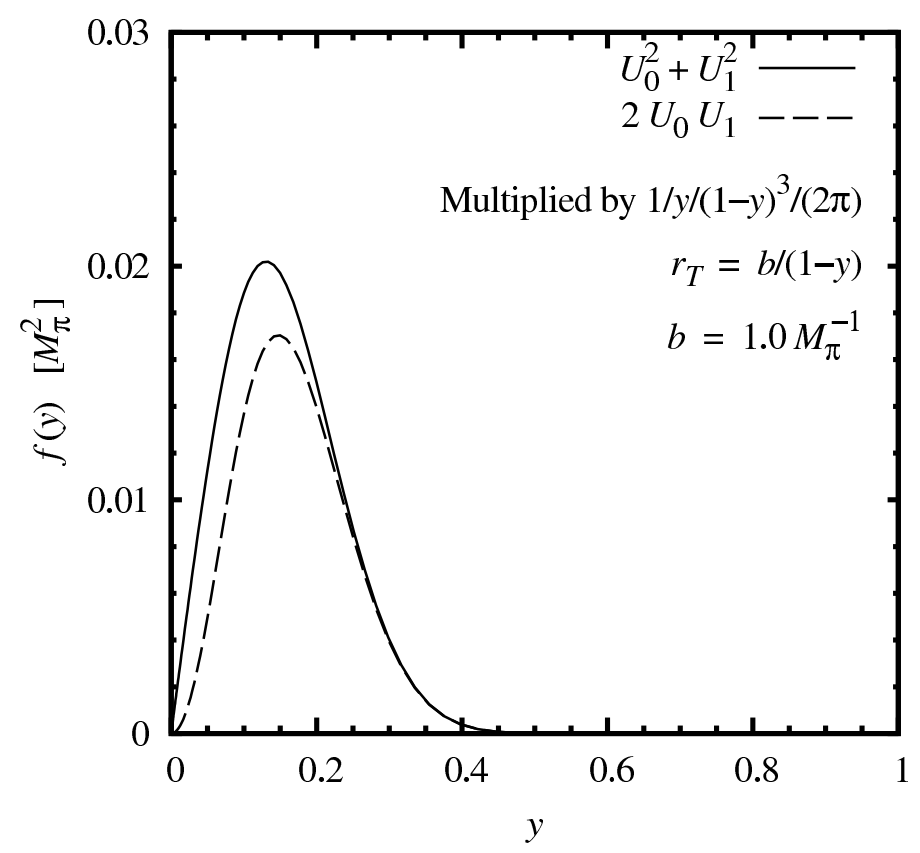

Figure 5. Integrands of the transverse densities $\rho_{1}^{V}$ and $-\widetilde{\rho}_{2}^{V}$ in the wave function overlap representation eq. (5.4), at a distance $b=1.0 M_{\pi}^{-1}$. The integrands are given in units of $M_{\pi}^{2}$.

the integrands are concentrated around values $y \sim M_{\pi} / M_{N}$. Contributions from $y>0.5$ are very strongly suppressed because the wave function is evaluated at separations $r_{T}=$ $b /(1-y)$ that are substantially larger than $b$, and the wave function decays exponentially at large $r_{T}$, with a range that itself decreases with increasing $y$. One also sees that the integrands for the densities $\rho_{1}^{V}$ and $-\widetilde{\rho}_{2}^{V}$ are close to each other at large values of $y$ and differ only at $y \rightarrow 0$, such that they are numerically close throughout the dominant region of integration. This follows from the similarity of the wave functions $U_{0}$ and $U_{1}$, cf. figure $4 \mathrm{a}$, and implies that $\rho_{1}^{V}(b) \approx-\widetilde{\rho}_{2}^{V}(b)$ at distances $b=$ few times $M_{\pi}^{-1}$.

The transverse densities obtained by performing the $y$-integral in eq. (5.4) are shown in figure 6. One sees that $\widetilde{\rho}_{2}^{V}<0$, and that the absolute value of the spin-dependent density is smaller than the spin-independent one, $\left|\widetilde{\rho}_{2}^{V}\right|<\rho_{1}^{V}$, as required by eq. (5.7). The inequality is almost saturated at distances $b \sim 1 M_{\pi}^{-1}$, as suggested by the integrands shown in figure 5 , but at larger distances $\left|\widetilde{\rho}_{2}^{V}\right|$ becomes significantly smaller than $\rho_{1}^{V}$. We note that the numerical densities obtained from the wave function overlap representation eq. (5.4) exactly reproduce those calculated in the dispersive approach of ref. [8], which provides a test of the calculational procedures. ${ }^{5}$

\subsection{Transverse polarization}

Further insight into the peripheral transverse densities can be gained by considering the case of transversely polarized nucleon states. Transverse polarization naturally explains the

\footnotetext{
${ }^{5}$ In figure 8 of ref. [8] the function represented by the dashed line is $-\widetilde{\rho}_{2}(b)$, not $\widetilde{\rho}_{2}(b)$ (the plot is labeled incorrectly). The dispersive calculation gives $\widetilde{\rho}_{2}(b)<0$, as does the wave function representation of the present work.
} 


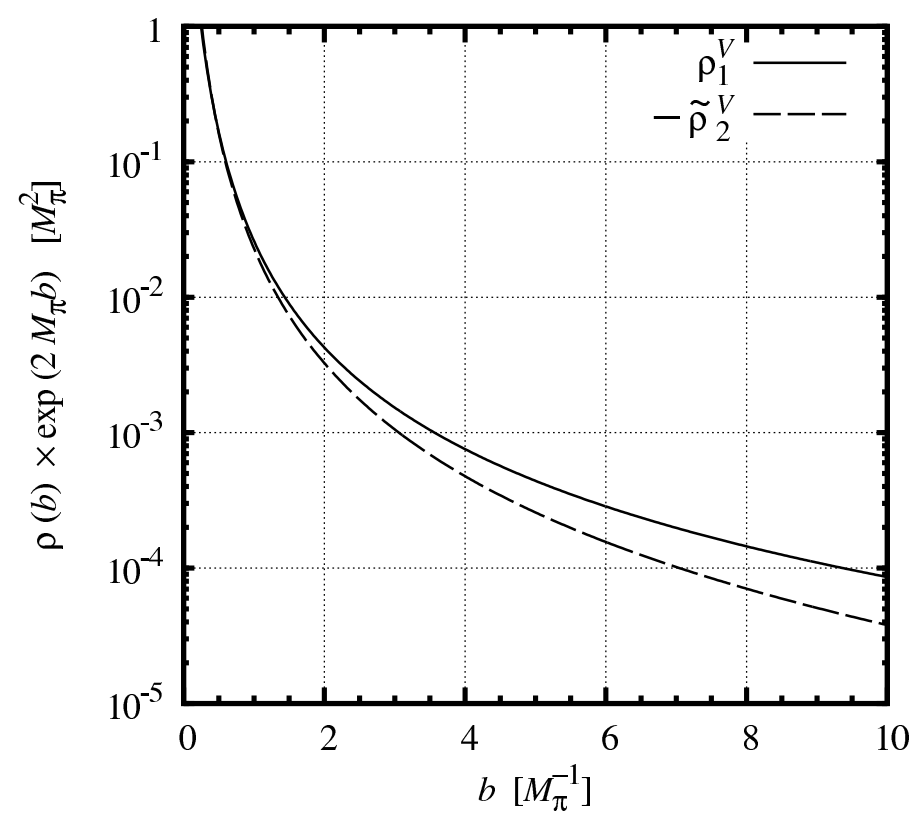

Figure 6. LO chiral component of the nucleon's isovector spin-independent current density $\rho_{1}^{V}(b)$ (solid line) and spin-dependent current density $-\widetilde{\rho}_{2}^{V}(b)$ (dashed line). The plot shows the densities with the exponential factor $\exp \left(-2 M_{\pi} b\right)$ extracted (the functions plotted correspond to the preexponential factor in eq. (3.1)).

similarity of the spin-independent and -dependent densities, $\rho_{1}^{V}(b)$ and $\widetilde{\rho}_{2}^{V}(b)$, at distances $b \sim$ few $M_{\pi}^{-1}$ (cf. figure 6) and enables a simple quantum-mechanical interpretation in the nucleon rest frame.

Transversely polarized nucleon states in the light-front formulation are obtained by preparing a transversely polarized state (say, in the $y$-direction) in the rest frame and performing the longitudinal and transverse boosts to the desired light-front momentum (cf. section 4.1). With the general formula eq. (4.1) this is accomplished simply by choosing the rest-frame 2-spinors as eigenspinors of the $y$-spin operator $S^{y}=\sigma^{y} / 2$,

$$
\chi_{\operatorname{tr}}(\tau=1 / 2)=\frac{1}{\sqrt{2}}\left(\begin{array}{l}
1 \\
i
\end{array}\right), \quad \chi_{\operatorname{tr}}(\tau=-1 / 2)=\frac{1}{\sqrt{2}}\left(\begin{array}{l}
i \\
1
\end{array}\right)
$$

(here and in the following we use $\tau= \pm 1 / 2$ to denote the $y$-spin eigenvalues). The vertex function calculated with the transversely polarized 4-spinors obtained in this way is of the same form as eq. (4.3); only the structures $S^{z}$ and $\boldsymbol{S}_{T}=\left(S^{x}, S^{y}\right)$ are replaced by the contraction of the spin operators with the transversely polarized spinors

$$
S_{\mathrm{tr}}^{i}\left(\tau, \tau_{1}\right) \equiv \chi_{\mathrm{tr}}^{\dagger}(\tau)\left(\frac{1}{2} \sigma^{i}\right) \chi_{\mathrm{tr}}\left(\tau_{1}\right) \quad(i=x, y, z) .
$$

Notice that now the $y$-component is diagonal,

$$
S_{\mathrm{tr}}^{y}\left(\tau, \tau_{1}\right)=\tau \delta\left(\tau, \tau_{1}\right),
$$

while the $z$ and $x$ components have off-diagonal terms. We define the light-front wave function for the $N \rightarrow \pi N$ transition for nucleon states characterized by their transverse 
spin projections $\tau_{1}$ and $\tau$ in complete analogy to eqs. (4.7)-(4.9),

$$
\begin{aligned}
\Psi_{\operatorname{tr}}\left(y, \widetilde{\boldsymbol{k}}_{T} ; \tau, \tau_{1}\right) & \equiv \frac{\Gamma_{\operatorname{tr}}\left(y, \widetilde{\boldsymbol{k}}_{T} ; \tau, \tau_{1}\right)}{\Delta \mathcal{M}^{2}\left(y, \widetilde{\boldsymbol{k}}_{T}\right)}, \\
\Gamma_{\operatorname{tr}}\left(y, \widetilde{\boldsymbol{k}}_{T} ; \tau, \tau_{1}\right) & =\frac{2 i g_{A} M_{N}}{F_{\pi} \sqrt{\bar{y}}}\left[y M_{N} S_{\mathrm{tr}}^{z}\left(\tau, \tau_{1}\right)+\widetilde{\boldsymbol{k}}_{T} \cdot \boldsymbol{S}_{\mathrm{tr}, T}\left(\tau, \tau_{1}\right)\right] .
\end{aligned}
$$

Similar expressions are obtained for the $\pi N \rightarrow N$ with transverse spin projections $\tau$ and $\tau_{2}$.

The coordinate-space wave functions for transverse nucleon polarization are introduced through eq. (4.11) in the same way as for longitudinal polarization and denoted by $\Phi_{\text {tr. }}$. The general decomposition eq. (4.12) applies to the Fourier transform of the transversely polarized wave function as well, as it relies only on the functional dependence of the momentum-space wave function on $\widetilde{\boldsymbol{k}}_{T}$, not on the specific form of the spin structures. Using the algebraic relation between the spin structures for $y$ - and $z$-polarization it is straightforward to express the transversely polarized coordinate-space wave function in terms of the longitudinally polarized radial wave functions $U_{0}$ and $U_{1}$ :

$$
\begin{aligned}
& \Phi_{\operatorname{tr}}\left(y, \boldsymbol{r}_{T}, \tau=+1 / 2, \tau_{1}=+1 / 2\right)=\sin \alpha U_{1}, \\
& \Phi_{\operatorname{tr}}\left(y, \boldsymbol{r}_{T}, \tau=-1 / 2, \tau_{1}=-1 / 2\right)=-\sin \alpha U_{1}, \\
& \Phi_{\operatorname{tr}}\left(y, \boldsymbol{r}_{T}, \tau=+1 / 2, \tau_{1}=-1 / 2\right)=U_{0}+\cos \alpha U_{1}, \\
& \Phi_{\operatorname{tr}}\left(y, \boldsymbol{r}_{T}, \tau=-1 / 2, \tau_{1}=+1 / 2\right)=-U_{0}+\cos \alpha U_{1}, \\
& \boldsymbol{r}_{T}=\left(r_{T} \cos \alpha, r_{T} \sin \alpha\right), \quad U_{0,1} \equiv U_{0,1}\left(y, r_{T}\right) .
\end{aligned}
$$

With the radial wave function $U_{0}$ and $U_{1}$ given by the explicit expressions of eq. (4.15), these relations completely determine the transversely polarized coordinate-space wave function.

In the transversely polarized representation the rotational symmetry around the $z$ axis is encoded in relations between the transverse spin components of the wave function. In the explicit formulas eqs. (5.14)-(5.17) these relations manifest themselves in that the four transverse spin components are expressed in terms of only two independent radial functions. One sees that the components satisfy

$$
\begin{aligned}
& \Phi_{\operatorname{tr}}\left(y, \boldsymbol{r}_{T},+,+\right)=-\Phi_{\operatorname{tr}}\left(y, \quad \boldsymbol{r}_{T},-,-\right), \\
& \Phi_{\operatorname{tr}}\left(y, \boldsymbol{r}_{T},+,-\right)=-\Phi_{\operatorname{tr}}\left(y,-\boldsymbol{r}_{T},-,+\right) .
\end{aligned}
$$

The relation eq. (5.19) between the transverse spin-flip components has a simple physical interpretation in the nucleon rest frame. In the wave function with initial nucleon transverse spin $\tau_{1}=+1 / 2$ and intermediate nucleon spin $\tau=-1 / 2$, the pion in the intermediate state has orbital angular momentum $L=1$ with projection $L^{y}=+1$ on the $y$-axis. Likewise, in the wave function with $\tau_{1}=-1 / 2$ and $\tau=+1 / 2$, the pion has $L^{y}=-1$. The two components thus differ only in that the pion rotates in the opposite sense around the $y$ axis, and one can be turned into the other by inverting the direction of the $x$-axis, i.e., replacing $\cos \alpha \rightarrow-\cos \alpha$ in eqs. (5.16) and (5.17).

The connection between the transversely and longitudinally polarized light-front wave functions generally depends on the angle of the transverse coordinate vector $\boldsymbol{r}_{T}$, as required 
by rotational invariance. A particularly simple connection is obtained at points on the negative or positive $x$-axis, where $\sin \alpha=0$ and $\cos \alpha=-1$ or +1 . Considering the transverse spin-flip wave function on the negative and positive $x$-axis ("left" and "right" when looking at the nucleon in the $z$-direction from $+\infty$, see figure 1 ), and introducing the short-hand notation

$$
\begin{aligned}
U_{\text {left }}\left(y, r_{T}\right) & \equiv \Phi_{\operatorname{tr}}\left(y,-r_{T} \boldsymbol{e}_{x}, \tau=-1 / 2, \tau_{1}=+1 / 2\right), \\
U_{\text {right }}\left(y, r_{T}\right) & \equiv \Phi_{\operatorname{tr}}\left(y,+r_{T} \boldsymbol{e}_{x}, \tau=-1 / 2, \tau_{1}=+1 / 2\right),
\end{aligned}
$$

we obtain from eq. (5.17)

$$
\begin{aligned}
U_{\text {left }}\left(y, r_{T}\right) & =-U_{0}\left(y, r_{T}\right)-U_{1}\left(y, r_{T}\right), \\
U_{\text {right }}\left(y, r_{T}\right) & =-U_{0}\left(y, r_{T}\right)+U_{1}\left(y, r_{T}\right) .
\end{aligned}
$$

Note that the functions $U_{\text {left }}$ and $U_{\text {right }}$ are derived from a single $y$-spin component of the wave function but refer to a specific spatial direction.

We can now derive a simple representation of the transverse densities in terms of the transversely polarized light-front wave function. Using the overlap representation eq. (5.4) in terms of the the longitudinally polarized wave functions, and substituting them by the "left" and "right" transverse spin-flip wave functions according to eqs. (5.22) and (5.23), we obtain ${ }^{6}$

$$
\left.\begin{array}{l}
\rho_{1}^{V}(b) \\
\widetilde{\rho}_{2}^{V}(b)
\end{array}\right\}=\frac{1}{4 \pi} \int \frac{d y}{y \bar{y}^{3}}\left\{\begin{array}{c}
{\left[U_{\text {left }}(y, b / \bar{y})\right]^{2}+\left[U_{\text {right }}(y, b / \bar{y})\right]^{2}} \\
-\left[U_{\text {left }}(y, b / \bar{y})\right]^{2}+\left[U_{\text {right }}(y, b / \bar{y})\right]^{2}
\end{array}\right\} .
$$

This result can be explained easily by referring to the interpretation of the transverse densities as current matrix elements in a nucleon state localized in transverse space, cf. section 2, figure 1, and ref. [8]. According to eq. (2.6), in a nucleon state with $y$-spin projection $+1 / 2$ (i.e., $\tau_{1}=\tau_{2}=+1 / 2$ ) the dependence of the matrix element on the coordinate $\boldsymbol{b}$ is of the form

$$
\left\langle J^{+}(\boldsymbol{b})\right\rangle_{\text {localized }}^{V}=(\ldots)\left[\rho_{1}^{V}(b)+\cos \phi \widetilde{\rho}_{2}^{V}(b)\right]
$$

where $\phi$ is the angle of $\boldsymbol{b}$ relative to the $x$-axis. The densities are functions of $b=|\boldsymbol{b}|$, and the angular dependence is given entirely by $\cos \phi$. By choosing the direction of $\boldsymbol{b}$ along the positive and negative $x$-axis $(\cos \phi= \pm 1)$, the individual densities can be expressed as

$$
\begin{aligned}
\left\langle J^{+}\left(b \boldsymbol{e}_{x}\right)+J^{+}\left(-b \boldsymbol{e}_{x}\right)\right\rangle_{\text {localized }}^{V} & =(\ldots) \rho_{1}^{V}(b), \\
\left\langle J^{+}\left(b \boldsymbol{e}_{x}\right)-J^{+}\left(-b \boldsymbol{e}_{x}\right)\right\rangle_{\text {localized }}^{V} & =(\ldots) \tilde{\rho}_{2}^{V}(b) .
\end{aligned}
$$

\footnotetext{
${ }^{6}$ For simplicity we derive the representation eq. (5.24) from eq. (5.4), using the relation between the longitudinally and transversely polarized wave functions at the special points $b= \pm b \boldsymbol{e}_{x}$. Equation (5.24) could equivalently be derived by converting the original current matrix element eq. (5.1) to the transverse spin representation and repeating the steps of section 5.1. In the latter approach one could choose any orientation of the vector $\boldsymbol{b}$; the rotational invariance of the densities would be guaranteed by the conditions eqs. (5.18) and (5.19).
} 


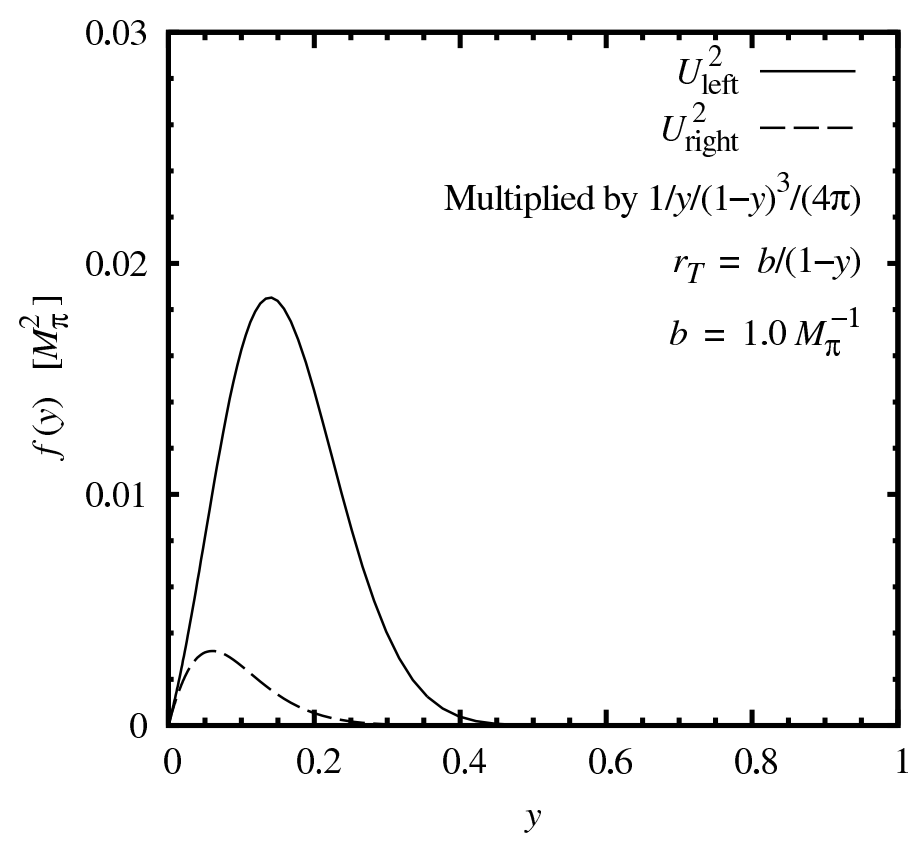

Figure 7. Integrands of the transverse densities $\rho_{1}^{V}$ and $\widetilde{\rho}_{2}^{V}$ in the transversely polarized wave function overlap representation eq. (5.24), at a distance $b=1.0 M_{\pi}^{-1}$. Solid line: $\left[U_{\text {left }}(y, b / \bar{y})\right]^{2}$; dashed line: $\left[U_{\text {right }}(y, b / \bar{y})\right]^{2}$. The functions are plotted including the factor $1 /\left(4 \pi y \bar{y}^{3}\right)$ and are given in units of $M_{\pi}^{2}$.

This structure is exactly analogous to eq. (5.24), with the integral over $\left|U_{\text {left }}(y, b / \bar{y})\right|^{2}$ and $\left|U_{\text {left }}(y, b / \bar{y})\right|^{2}$ representing the current densities at $b= \pm b \boldsymbol{e}_{x}$, respectively.

The explicit form of the "left" and "right" transverse spin-flip wave function, eqs. (5.20) and (5.21), is readily obtained from eqs. (5.22), (5.23) and (4.15):

$$
\begin{aligned}
& \left.\begin{array}{r}
U_{\text {left }}\left(y, r_{T}\right) \\
U_{\text {right }}\left(y, r_{T}\right)
\end{array}\right\}=-\frac{g_{A} M_{N} y \sqrt{\bar{y}}}{2 \pi F_{\pi}}\left\{\begin{array}{l}
{\left[y M_{N} K_{0}\left(M_{T} r_{T}\right)+M_{T} K_{1}\left(M_{T} r_{T}\right)\right]} \\
{\left[y M_{N} K_{0}\left(M_{T} r_{T}\right)-M_{T} K_{1}\left(M_{T} r_{T}\right)\right]}
\end{array}\right\} \\
& \sim-\frac{g_{A} M_{N} y \sqrt{\bar{y}}}{2 \sqrt{2 \pi} F_{\pi}}\left\{\begin{array}{l}
\left(y M_{N}+M_{T}\right) \\
\left(y M_{N}-M_{T}\right)
\end{array}\right\} \frac{e^{-M_{T} r_{T}}}{\sqrt{M_{T} r_{T}}} \quad\left(r_{T} M_{T} \gg 1\right) .
\end{aligned}
$$

The last expression is obtained with the asymptotic form of the modified Bessel functions, eq. (4.16), and applies at $M_{T} r_{T} \gg 1$. Parametrically the two functions are of the same order in $M_{\pi} / M_{N}$ in the region $y=O\left(M_{\pi} / M_{N}\right)$ and $r_{T}=O\left(M_{\pi}^{-1}\right)$. Numerically one observes that

$$
\left|U_{\text {left }}\left(y, r_{T}\right)\right| \gg\left|U_{\text {right }}\left(y, r_{T}\right)\right| \quad\left(y \sim \text { few times } M_{\pi} / M_{N}\right)
$$

because $M_{T}(y) \approx y M_{N}$ in this region of $y$. This is illustrated by figure 7 , which shows the contributions of $\left|U_{\text {left }}\right|^{2}$ and $\left|U_{\text {right }}\right|^{2}$ to the integrands of the transverse densities in eq. (5.24). The strong suppression of the "right" compared to the "left" wave function is the reason for the similarity of the densities $\rho_{1}^{V}(b)$ and $-\widetilde{\rho}_{2}^{V}(b)$ at $b=O\left(M_{\pi}^{-1}\right)$. 


\subsection{Quantum-mechanical picture}

We can interpret our findings in a simple quantum-mechanical picture of peripheral transverse nucleon structure in the rest frame. For this purpose we imagine that the $N \rightarrow \pi N$ transition takes place in ordinary time, and that the wave function has the usual 3dimensional rotational symmetry. For a non-relativistic system there would be a direct correspondence between the equal-time and the light-front wave functions; see e.g. ref. [28]. The chiral $\pi N$ system is essentially relativistic, $k=O\left(M_{\pi}\right)$, and one should not expect a similar connection between the wave functions here. Nevertheless the intuitive quantummechanical picture of the chiral process explains all the essential features of the peripheral transverse densities. In any case its content is backed up by light-front wave function formulas, which are exact also in the relativistic case.

Consider a nucleon in the rest frame, in a spin state polarized in the positive $y$ direction $\left(S^{y}=+1 / 2\right)$. In the interaction picture implied by chiral EFT, we may think of this physical nucleon as a pointlike bare nucleon coupled to soft pions, described by a wave function. The LO contribution to the peripheral charge and current densities in the proton isospin state arises from the component with a single peripheral positively charged pion. This component corresponds to the chiral process where the initial (bare) proton makes a transition to a state with a (bare) neutron and a peripheral positive pion, and back to the final bare nucleon (see figure 8). The $y$-spin projection quantum numbers of the initial/final nucleon state are $\tau_{1}=\tau_{2}=+1 / 2$, and that of the intermediate nucleon state is denoted by $\tau$. Because of parity conservation the wave function of the $\pi^{+} n$ system has orbital angular momentum $L=1$. Conservation of the total angular momentum projection on the $y$-axis allows only the states with $L^{y}=0$ and $\tau=+1 / 2$ (spin-conserving), and with $L^{y}=+1$ and $\tau=-1 / 2$ (spin-flip). We are interested in the densities in the $x$ - $z$ plane $(y=0)$, to which the state with $L^{y}=0$ cannot contribute, as its wave function vanishes in the direction perpendicular to the $y$-axis: $P_{1}(\cos \theta)=0$ for $\theta=\pi / 2\left(P_{1}\right.$ is the Legendre polynomial of degree 1). This leaves the state with $L^{y}=+1$ and $\tau=-1 / 2$ as the only contribution to the densities in question. It explains why in the light-front formulation we were able to express the transverse densities completely in terms of the transverse spin-flip wave function, cf. eqs. (5.20) and (5.21).

The peripheral densities in the $x-z$ plane thus arise from configurations in which the positive pion "orbits" around the neutron with angular momentum $L^{y}=+1$. Since the pion itself has no spin, the current it produces is the convection current caused by the orbital motion of the charge. Because the chiral EFT interactions between the pion and the nucleon have a short range $\ll M_{\pi}^{-1}$, the peripheral pion can be regarded as free while the current is measured. The 4 -vector current carried by a free pion with momentum $\boldsymbol{k}$ and charge +1 is $J^{\mu}=2 k^{\mu}$, where $k^{0} \equiv E_{\pi}=\sqrt{|\boldsymbol{k}|^{2}+M_{\pi}^{2}}$, so that its plus component is positive for all momenta, $J^{+}=2 k^{+}=2\left(E_{\pi}+k^{z}\right)>0$. This explains the positivity property of the current density, eq. (5.8).

Now according to section 2 and eq. (2.6) the transverse densities $\rho_{1}^{V}$ and $\widetilde{\rho}_{2}^{V}$ are the left-right average and left-right asymmetry of the $J^{+}$current density produced by the peripheral pion. It is obvious that a positively charged pion orbiting with $L^{y}=+1$ produces 


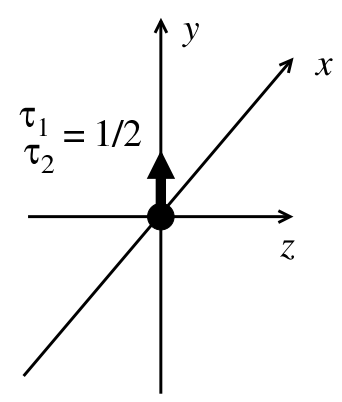

initial/final state

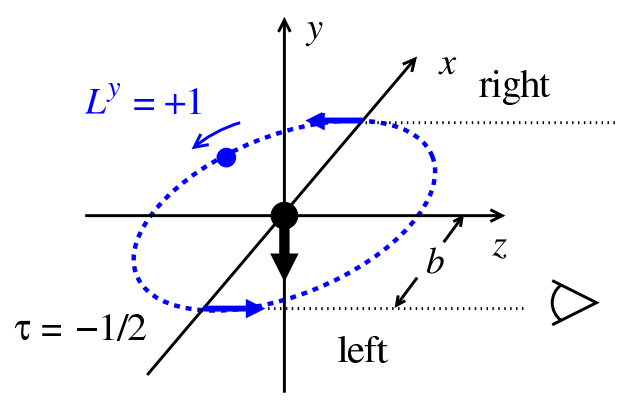

intermediate state

Figure 8. Quantum-mechanical picture of peripheral transverse densities in LO chiral EFT. The bare nucleon with $y$-spin projection $\tau_{1}=+1 / 2$ in the rest frame (left) makes a transition to a pionnucleon state with $\tau=-1 / 2$ and $L^{y}=+1$ (right), and back to a bare state with $\tau_{2}=+1 / 2$. The peripheral transverse densities $\rho_{1}^{V}(b)$ and $\widetilde{\rho}_{2}^{V}(b)$ are the left-right average and left-right asymmetry of the plus current (viewed from $z=+\infty$ ) at the positions $y=0$ and $x=\mp b$.

a larger $J^{+}$density on the left (where it moves in the positive $z$ direction) than on the right (where it moves in the negative $z$ direction). This explains why $\widetilde{\rho}_{2}^{V}(b)<0$ (see figure 6 ). The magnitude of the asymmetry is determined by the effective pion momenta and the relativistic effects implied by the projection on fixed light-front time. If the motion of the pion were non-relativistic, with characteristic velocity $v=k / M_{\pi} \ll 1$, the plus momentum carried by the pion would be $k^{+}=M_{\pi}[1+O(v)]$; i.e., it would be dominated by the pion mass and independent of the direction of the pion momentum. Since furthermore the probability to find a pion would be the same on the left and on the right side of the $x$ axis (because of rotational symmetry around the $y$-axis), the ratio of left and right current densities at the same distance $b=|\boldsymbol{b}|$ would be

$$
\frac{\left\langle J^{+}\left(-b \boldsymbol{e}_{x}\right)\right\rangle_{\text {localized }}}{\left\langle J^{+}\left(+b \boldsymbol{e}_{x}\right)\right\rangle_{\text {localized }}}=1+O(v) .
$$

The light-front wave function representation of section 5.4 shows that the asymmetry is much larger than the non-relativistic estimate eq. (5.31),

$$
\frac{\left\langle J^{+}\left(-b \boldsymbol{e}_{x}\right)\right\rangle_{\text {localized }}}{\left\langle J^{+}\left(+b \boldsymbol{e}_{x}\right)\right\rangle_{\text {localized }}}=\frac{\int \frac{d y}{y \bar{y}^{3}}\left|U_{\text {left }}(y, b / \bar{y})\right|^{2}}{\int \frac{d y}{y \bar{y}^{3}}\left|U_{\text {right }}(y, b / \bar{y})\right|^{2}} \gg 1
$$

The numerical value of the ratio is $\sim 9$ at $b=1 M_{\pi}^{-1}$ and $\sim 4$ at $b=5 M_{\pi}^{-1}$ (see figure 7 ). This highlights the essentially relativistic nature of the motion of pions in chiral dynamics. The power of the light-front formulation is that it permits a first-quantized representation even of such essentially relativistic systems.

\subsection{Contact term}

The transverse charge density $\rho_{1}^{V}(b)$ also receives a contribution from the effective contact term in the current matrix element, eq. (3.9). This contribution cannot be represented as 


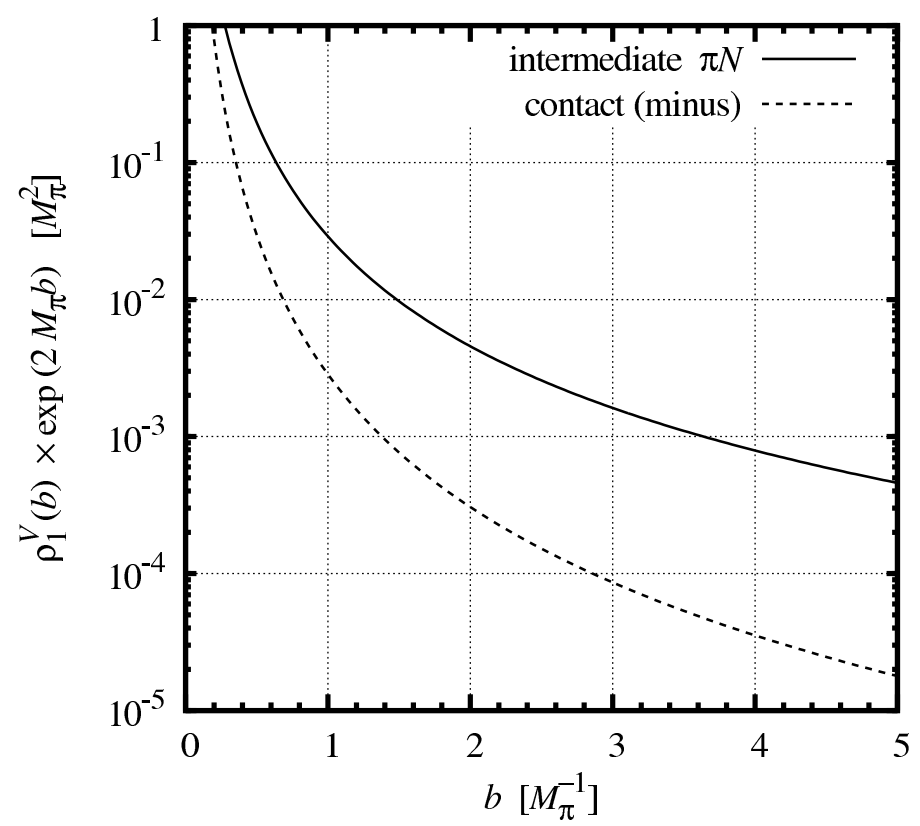

Figure 9. LO chiral component of the nucleon's isovector spin-independent current density $\rho_{1}^{V}(b)$. Solid line: contribution from intermediate $\pi N$ states, eqs. (3.8) and (5.4). Dotted line: effective contact term, eq. (3.9), plotted with opposite sign (the actual contribution is negative). The plot shows the contributions to the density with the exponential factor $\exp \left(-2 M_{\pi} b\right)$ extracted (the functions plotted correspond to the pre-exponential factor in eq. (3.1)).

an overlap of $\pi N$ light-front wave functions and needs to be added separately to that from the intermediate $\pi N$ state, eq. (5.4),

$$
\left.\rho_{1}^{V}(b)=\rho_{1}^{V}(b)_{\text {interm }} \text { [from eq. }(5.4)\right]+\rho_{1}^{V}(b)_{\text {contact }} .
$$

It is readily computed by evaluating the Feynman integral eq. (3.9) as a four-dimensional integral, using the fact that it depends only on the momentum transfer $\Delta^{\mu}$ as external 4 -vector. One obtains [8]

$$
\rho_{1}^{V}(b)_{\text {contact }}=\frac{\left(1-g_{A}^{2}\right) M_{\pi}^{4}}{192 \pi^{3} F_{\pi}^{2}}\left\{\left[K_{2}\left(M_{\pi} b\right)\right]^{2}-4\left[K_{1}\left(M_{\pi} b\right)\right]^{2}+3\left[K_{0}\left(M_{\pi} b\right)\right]^{2}\right\}
$$

The contact term density is negative because $1-g_{A}^{2}<0$. It is of the same parametric order as the one from intermediate $\pi N$ states, as can be seen by comparing eq. (5.34) with eqs. (5.4) and (4.15) and noting that at distances $b=O\left(M_{\pi}^{-1}\right)$ the integral is dominated by pion momentum fractions $y=O\left(M_{\pi} / M_{N}\right)$. The numerical contribution of the contact term density turns out to be very small in the region of interest, $b=$ few $M_{\pi}^{-1}$, ranging from about $-10 \%$ of the intermediate $\pi N$ contributions at $b=1 M_{\pi}^{-1}$ to $-4 \%$ at $b=5 M_{\pi}^{-1}$ (see figure 9). Thus the entire $\rho_{1}^{V}$ is to good approximation given by the wave function overlap eq. (5.4), which justifies our earlier comparison of the properties of $\rho_{1}^{V}$ and $\widetilde{\rho}_{2}^{V}$ on the basis of eq. (5.4) (the contact term is absent in $\widetilde{\rho}_{2}^{V}$ ).

Some comments are in order regarding the interpretation of the contact term in the context of the light-front description. In the intermediate $\pi N$ contribution to the current 
matrix element the typical energy denominators are $\Delta \mathcal{M}^{2}\left(y, \boldsymbol{k}_{T}, \boldsymbol{p}_{1 T, 2 T}\right)=O\left(M_{\pi} M_{N}\right)$, cf. eqs. (3.18) and (3.19), and the typical pion light-front energies in the nucleon rest frame are $k_{1,2}^{-}=O\left(M_{\pi}\right)$. These are configurations that exist for a large light-front time interval $\Delta x^{-}=O\left(M_{\pi}^{-1}\right)$ and can be regarded as particle states in the chiral EFT. The contact term describes contributions to the peripheral density from intermediate states with invariant mass differences that are not chirally small, i.e., that do not vanish in the limit $M_{\pi} \rightarrow 0$. These states lie "outside" the chiral EFT, and their contribution is represented by a local operator.

The appearance of the combination $1-g_{A}^{2}$ in the coefficient of the local operator is natural [8]. For a nucleon without internal structure one would have $g_{A}=1$, and the effective contact term would be absent. The deviation of $g_{A}$ from unity is the result of the "compositeness" of the nucleon, which in turn is related to the presence of inelastic states in $\pi N$ scattering. The combination $1-g_{A}^{2}$ can thus be regarded as the effect of nonchiral intermediate states in the $\pi N$ scattering amplitude, in agreement with the above interpretation. ${ }^{7}$ Further properties of the contact term, such as its relation to the form of the $\pi N N$ coupling (pseudoscalar, axial vector) are discussed in ref. [8].

The contact term formally corresponds to a contribution of light-front zero modes (vanishing pion plus momentum) to the current matrix element. An advantage of our approach, starting from the invariant formulation of chiral EFT, is that it allows us to identify and calculate these contributions in a straightforward manner. The zero mode contributions could also be calculated in the time-ordered formulation of chiral EFT, by considering the current matrix in a frame where the current transfers plus momentum, $\Delta^{+} \neq 0$, and taking the limit $\Delta^{+} \rightarrow 0$ at the end of the calculation.

\subsection{Region of applicability}

To conclude our study of the peripheral transverse densities we want to comment on the region of applicability of chiral dynamics and the comparison of our results with empirical transverse densities. These questions have been investigated in detail in refs. [8, 20,31], and we only summarize the essential points here.

The region of distances where the nucleon's transverse densities are described by chiral dynamics can be quantified using the dispersive representation, in which the densities are expressed as integrals of the imaginary parts (or spectral functions) of the nucleon's elastic form factors along the cut at $t>4 M_{\pi}^{2}[20,31]$. Chiral dynamics governs the isovector spectral functions in the near-threshold region $t=4 M_{\pi}^{2}+$ few $M_{\pi}^{2}$ (the LO chiral contribution to the spectral functions is given precisely by the cut diagrams of figure 2a). At larger values $t \lesssim 1 \mathrm{GeV}^{2}$ the spectral functions are dominated by the $\rho$ meson resonance; at even larger $t$ they are composed from a continuum of higher-mass exchanges. The dispersive representation allows one to determine the relative contributions of these $t$ regions to the transverse densities at a given distance $b$. Numerical studies find that the chiral contributions become significant only at $b \gtrsim 2 \mathrm{fm}$ and dominate the total densities

\footnotetext{
${ }^{7}$ The connection between $g_{A}^{2}-1$ and inelastic states in $\pi N$ scattering is expressed in general terms by the Adler-Weisberger current algebra sum rule [29, 30].
} 
at $b \gtrsim 3 \mathrm{fm}$ [31]. At such distances the nucleon's charge and magnetization densities are governed by chiral dynamics, and the mechanical picture of peripheral non-interacting pions developed in section 5.5 is applicable. At distances $b \lesssim 2 \mathrm{fm}$ the densities are generated mostly by the $\rho$ meson mass region of the dispersive integral and thus involve short-range interactions beyond chiral dynamics. Whether the EFT-based description of the transverse densities could be extended to such distances, with a suitable finite-size generalization of the $\pi N N$ vertex and the pion form factor, is an interesting question for further study.

The dispersive representation also allows us to compare our results with empirical transverse densities and assess to what extent the peripheral region is constrained by present form factor data. This representation respects the analytic properties of the nucleon form factors in $t$, which is essential for extracting the peripheral transverse densities $[20,31]{ }^{8}$ Fits to the nucleon form factor data have been performed in the dispersive representation $[32,33]$ and describe the available data well. In these fits the spectral functions above the threshold at $t=4 M_{\pi}^{2}$ (which is in the unphysical region) have to be supplied as a theoretical input and are calculated using dispersion theory [34]. Chiral EFT in the LO approximation reproduces the dispersion theory results for the spectral functions near threshold, with a discrepancy that is zero at threshold (soft-pion limit) and reaches $\sim 1 / 2$ of the dispersion result at $t=10 M_{\pi}^{2}$ for $\operatorname{Im} F_{1}(t)$, and $\sim 3 / 4$ for $\operatorname{Im} F_{2}(t)$; see refs. [27, 31] for plots. The spectral functions in this region determine the peripheral transverse densities at distances $b \gtrsim 3 \mathrm{fm}$ [31]. We conclude that our LO chiral densities, eqs. (5.4) and (5.24), reproduce the empirical transverse densities from dispersive fits to form factor data exactly in the limit $b \rightarrow \infty$, and with a relative discrepancy significantly less than $1 / 2$ for the charge density at $b \sim 3 \mathrm{fm}$, and significantly less than $3 / 4$ for the magnetization density. Note that the peripheral densities are discussed on a logarithmic scale, so that such agreement is quite reasonable. Next-to-leading order (NLO) chiral corrections reduce the discrepancies in the near-threshold spectral functions (and thus in the peripheral densities) by roughly a factor of 2 [27] and could be incorporated into our light-front wave function description of the peripheral densities. We emphasize that the present form factor data at $t<0$ are only marginally sensitive to the spectral functions near threshold, and do not test them with a precision comparable to that of the dispersion theory calculation, so that the spectral functions remain an essentially theoretical input to the dispersive form factor fits. In this sense the comparison between "empirical" and "chiral" densities performed here is really a theory-to-theory comparison between dispersion theory and chiral EFT. To what extent future precision measurements of the form factors at low momentum tranfers $|t| \ll 0.01 \mathrm{GeV}^{2}$ could experimentally test the spectral functions of the form factors near

\footnotetext{
${ }^{8}$ The transverse densities $\rho_{1,2}(b)$ are the Fourier transforms of the invariant form factors $F_{1,2}\left(t=-\boldsymbol{\Delta}_{T}^{2}\right)$ with respect to the transverse momentum transfer $\boldsymbol{\Delta}_{T}$, i.e., the inverse of the Fourier representation eq. (2.5). The asymptotic behavior of the densities at large $b$ is determined by the singularities of the form factors as complex functions of $t$. The physical form factors have a principal cut starting at $t=4 M_{\pi}^{2}$ and running to $t \rightarrow+\infty$, and no other complex singularities on the physical sheet, which implies exponential fall-off of the transverse densities at $b \rightarrow \infty$, cf. eq. (3.1). The dispersive representation respects this analytic structure and gives densities with the correct asymptotic behavior. Form factor parametrizations with unphysical analytic properties, such as ratios of higher-order polynomials [7], generally produce densities with incorrect asymptotic behavior and cannot be used to extract peripheral densities [31].
} 
threshold, and in this way constrain the peripheral transverse densities, is an interesting question that calls for a dedicated study.

\section{Chiral generalized parton distributions}

\subsection{Peripheral pion distribution}

In QCD the transverse structure of the nucleon is expressed in terms of coordinatedependent parton densities, which are defined as the Fourier transforms of the generalized parton distributions and describe the density of partons (quarks, antiquarks, gluons) with a given light-front plus momentum fraction $x$ at transverse position $\boldsymbol{b}$. In this context the transverse charge and current densities are obtained as integrals of the transverse densities of charged partons (quarks minus antiquarks) over $x$. Chiral dynamics governs not only the peripheral charge and current densities but also the $x$-dependent distributions of partons at $b \equiv|\boldsymbol{b}|=O\left(M_{\pi}^{-1}\right)$ and $x=O\left(M_{\pi} / M_{N}\right)$. Detailed studies of the peripheral parton densities due to chiral dynamics have been performed in refs. [21, 22]. Here we show that the $x$-integral of these peripheral parton densities reproduces the transverse charge and current densities calculated in chiral EFT. We also express the plus momentum distribution of peripheral pions in the nucleon in terms of the $\pi N$ light-front wave functions introduced in section 3 .

The basic object in the study of peripheral partonic structure is the light-front plus momentum distribution of soft pions in the nucleon. Following refs. [21, 22] we define the GPDs of soft pions in the nucleon in terms of the matrix element of the bilinear light-ray operator in the pion field,

$$
\begin{aligned}
& p^{+} \int_{-\infty}^{\infty} \frac{d \xi^{-}}{2 \pi} e^{i y p^{+} \xi^{-} / 2} \\
& \times\left\langle N\left(p_{2}, \sigma_{2}\right)\left|\left[\sum_{a b} \epsilon^{3 a b} \pi^{a}(-\xi / 2) \stackrel{\leftrightarrow}{\partial}+\left.\pi^{b}(\xi / 2)\right|_{\xi^{+}=0, \xi_{T}=0}\right]\right| N\left(p_{1}, \sigma_{1}\right)\right\rangle \\
& =\bar{u}_{2}\left[\gamma^{+} H_{\pi}^{V}(y, t)-\frac{E_{\pi}^{V}(y, t)}{2 M_{N}} \sigma^{+\nu} \Delta_{\nu}\right] u_{1},
\end{aligned}
$$

where $\xi$ is the 4 -vector of the space-time separation of the fields and $\stackrel{\leftrightarrow}{\partial}^{\mu} \equiv\left(\vec{\partial}^{\mu}-\overleftarrow{\partial}^{\mu}\right) / 2$ Equation (6.1) applies in the parametric regime of pion momentum fractions $y=O\left(M_{\pi} / M_{N}\right)$ and momentum transfers $t=O\left(M_{\pi}^{2}\right)$ and is to be evaluated in chiral EFT. The pionic operator on the left-hand side has isovector quantum numbers, and the matrix element is understood in the sense of eq. (2.8). The GPDs are denoted by $H_{\pi}^{V}$ and $E_{\pi}^{V}$ in accordance with the standard convention [35], and are defined in the interval $-1<y<1$. It is easy to see that the pionic light-ray operator is symmetric under $\xi \rightarrow-\xi$, whence the isovector GPDs are even functions of $y$,

$$
H_{\pi}^{V}(y, t)=H_{\pi}^{V}(-y, t), \quad E_{\pi}^{V}(y, t)=E_{\pi}^{V}(-y, t) .
$$


Note that for $\xi=0$ the pionic operator reduces to the local vector current of the pion field,

$$
J^{+}(0)=\sum_{a b} \epsilon^{3 a b} \pi^{a}(0) \partial^{+} \pi^{b}(0),
$$

the matrix element of which determines the chiral $\pi \pi$ cut contribution to the Dirac and Pauli form factors and was calculated in section 3.1. When integrating eq. (6.1) over $y$ the exponential factor produces a delta function, which enforces $\xi^{-}=0$ and thus $\xi=0$, so that the matrix element of the light-ray operator becomes that of the local vector current. In this sense eq. (6.1) is just a particular representation, differential in the pion plus momentum fraction $y$, of the chiral $\pi \pi$ cut in the current matrix element.

The transverse coordinate-dependent (or impact parameter-dependent) distributions of soft pions in the nucleon are then defined in analogy to the transverse charge and current densities, eq. (2.5), as

$$
\left.\begin{array}{l}
H_{\pi}^{V}\left(y, t=-\boldsymbol{\Delta}_{T}^{2}\right) \\
E_{\pi}^{V}\left(y, t=-\boldsymbol{\Delta}_{T}^{2}\right)
\end{array}\right\}=\int d^{2} b e^{i \boldsymbol{\Delta}_{T} \boldsymbol{b}}\left\{\begin{array}{l}
f_{1 \pi}^{V}(y, b) \\
f_{2 \pi}^{V}(y, b)
\end{array}\right\} .
$$

It is convenient to introduce a modified helicity-flip distribution in analogy to $\widetilde{\rho}_{2}$, eq. (2.7),

$$
\widetilde{f}_{2 \pi}^{V}(y, b) \equiv \frac{\partial}{\partial b}\left[\frac{f_{2 \pi}^{V}(y, b)}{2 M_{N}}\right] .
$$

The functions $f_{1 \pi}^{V}(y, b)$ and $\widetilde{f}_{2 \pi}^{V}(y, b)$ describe the isovector transverse spatial distribution of pions with plus momentum fraction $y$ and refer to the parametric regime $b=O\left(M_{\pi}^{-1}\right)$ and $y=O\left(M_{\pi} / M_{N}\right)$. The interpretation of these spatial distributions in a transversely polarized nucleon is analogous to that of the transverse densities $\rho_{1}^{V}(b)$ and $\widetilde{\rho}_{2}^{V}(b)$, cf. eq. (2.6) and figure 1.

The peripheral pion distributions $f_{1 \pi}^{V}(y, b)$ and $\widetilde{f}_{2 \pi}^{V}(y, b)$ can be calculated in LO chiral EFT in the same manner as the transverse densities $\rho_{1}^{V}(b)$ and $\widetilde{\rho}_{2}^{V}(b)$, cf. section 3 . One expresses the matrix element in eq. (6.1) as a Feynman integral and separates it into the intermediate nucleon contribution and an effective contact term, cf. eqs. (3.7)-(3.9). The intermediate nucleon contribution can then be represented as the product of the lightfront wave functions describing the transition of the initial and final nucleon to the $\pi N$ intermediate state. For $y>0$,

$$
\left.\begin{array}{c}
f_{1 \pi}^{V}(y, b) \\
\widetilde{f}_{2 \pi}^{V}(y, b)
\end{array}\right\}=\frac{1}{2 \pi y \bar{y}^{3}}\left\{\begin{array}{c}
{\left[U_{0}(y, b / \bar{y})\right]^{2}+\left[U_{1}(y, b / \bar{y})\right]^{2}} \\
-2 U_{0}(y, b / \bar{y}) U_{1}(y, b / \bar{y})
\end{array}\right\} \quad(y>0),
$$

where $U_{0,1}$ are the coordinate-space wave functions defined in eqs. (4.12) and (4.15); for $y<0$ one uses that (cf. eq. (6.2))

$$
f_{1 \pi}^{V}(y, b)=f_{1 \pi}^{V}(-y, b), \quad \widetilde{f}_{2 \pi}^{V}(y, b)=\widetilde{f}_{2 \pi}^{V}(-y, b) .
$$

The contact term contribution is obtained from the Feynman integral as

$$
f_{1 \pi}^{V}(y, b)_{\text {contact }}=\delta(y) \rho_{1}^{V}(b)_{\text {contact }},
$$


where $\rho_{1}^{V}(b)_{\text {contact }}$ is given by eq. (5.34). This expression clearly identifies the contact term as a light-front "zero mode" contribution. The complete isovector pion distribution $f_{1 \pi}^{V}$ is then given by the sum of the overlap contribution eq. (6.6) and the contact term eq. (6.8). This representation of the chiral pion densities in particular implies that (cf. eqs. (5.4) and (5.33))

$$
\left.\begin{array}{l}
\rho_{1}^{V}(b) \\
\tilde{\rho}_{2}^{V}(b)
\end{array}\right\}=\int_{-1}^{1} d y\left\{\begin{array}{l}
f_{1 \pi}^{V}(y, b) \\
\widetilde{f}_{2 \pi}^{V}(y, b)
\end{array}\right\},
$$

as is obvious from the above definition of $f_{1 \pi}^{V}$ and $f_{2 \pi}^{V}$. In this sense our earlier results for the peripheral transverse densities in chiral EFT have a straightforward interpretation as $y$-integrals of the peripheral pion GPDs in the nucleon. The significance of this connection lies in the fact that the peripheral pion GPDs have a more general physical meaning and can in principle be measured independently in peripheral high-energy scattering processes.

\subsection{Charge density from peripheral partons}

We can now demonstrate the connection of the chiral component of the peripheral transverse densities with the peripheral quark/antiquark content of the nucleon in QCD. Following ref. [22] the isovector quark/antiquark density in the nucleon at $b=O\left(M_{\pi}^{-1}\right)$ generated by chiral dynamics is given by

$$
[u-d](x, b)_{\text {chiral }}=[\bar{d}-\bar{u}](x, b)_{\text {chiral }}=\int_{x}^{1} \frac{d y}{y} f_{1 \pi}^{V}(y, b) q_{\pi}^{\mathrm{val}}(z) \quad(z \equiv x / y),
$$

where $z=x / y$ represents the fraction of the pion plus momentum carried by the quark/ antiquark, and $q_{\pi}^{\mathrm{val}}(z)$ is the valence quark/antiquark density in the pion,

$$
q_{\pi}^{\mathrm{val}}(z)= \pm[\bar{d}-\bar{u}]_{\pi \pm}(z)= \pm[u-d]_{\pi \pm}(z)= \pm \frac{1}{2}[u-\bar{u}-d+\bar{d}]_{\pi \pm}(z)
$$

normalized such that

$$
\int_{0}^{1} d z q_{\pi}^{\mathrm{val}}(z)=1
$$

Equation (6.10) has the form of the usual partonic convolution formulas and relies on the approximation that the non-chiral transverse size of the pion can be neglected on the scale $O\left(M_{\pi}^{-1}\right)$, so that the spatial distribution of peripheral quarks/antiquarks is determined entirely by the distribution of pions in the nucleon. The transverse charge density in the proton is generally given by the integral of the quark minus antiquark densities over $x$, weighted by the quark charges $\left(e_{u}=2 / 3, e_{d}=-1 / 3\right)$,

$$
\rho_{1}^{p}(b)=\int_{0}^{1} d x\left\{e_{u}[u-\bar{u}](x, b)+e_{d}[d-\bar{d}](x, b)\right\} .
$$

The isovector density is obtained by taking half the proton-neutron difference and using isospin symmetry,

$$
\rho_{1}^{V}(b) \equiv \frac{1}{2}\left[\rho_{1}^{p}-\rho_{1}^{n}\right](b)=\frac{1}{2}\left(e_{u}-e_{d}\right) \int_{0}^{1} d x[u-\bar{u}-d+\bar{d}](x, b) \quad\left(e_{u}-e_{d}=1\right)
$$


(this expression is valid even when including strange quarks in the individual proton and neutron densities). Substituting here the peripheral quark/antiquark densities generated by chiral dynamics, eq. (6.10), and using the normalization condition eq. (6.12), one obtains

$$
\begin{aligned}
\rho_{1}^{V}(b)_{\text {chiral }} & =\int_{0}^{1} d x[u-\bar{u}-d+\bar{d}](x, b)_{\text {chiral }} \\
& =\int_{0}^{1} d x \int_{x}^{1} \frac{d y}{y} f_{1 \pi}^{V}(y, b) q_{\pi}^{\mathrm{val}}(x / y) \\
& =\int_{0}^{1} d y f_{1 \pi}^{V}(y, b) \int_{0}^{y} \frac{d x}{y} q_{\pi}^{\mathrm{val}}(x / y) \\
& =\int_{0}^{1} d y f_{1 \pi}^{V}(y, b)
\end{aligned}
$$

which agrees with eq. (6.9). It shows that resolving the peripheral pion into its quark/antiquark constituents and computing the charge density from the quark/antiquark densities in the nucleon leads to the same result as computing the charge density directly from the distribution of (pointlike) pions - as it should be in the parton picture. The same applies, of course, to the spin-dependent density $\widetilde{\rho}_{2}^{V}(b)$.

The $x \rightarrow 0$ limit in the integral eq. (6.14) and the role of the contact term in the charge density in the "sum rule" eq. (6.15) require special consideration. In the LO chiral expansion of the current matrix element and the correlator eq. (6.1) it is supposed that the pion plus momentum fraction is of the parametric order $y=O\left(M_{\pi} / M_{N}\right)$. The contributions from pions with $y \rightarrow 0$ on this scale are described by a delta function $\delta(y)$, i.e., one sees only their contribution to the total charge density ( $y$-integral, first moment) but cannot resolve their dependence on $y$. In this sense the LO chiral expression for the peripheral parton densities is valid for $x=O\left(M_{\pi} / M_{N}\right)$ but otherwise not exceptionally small. Now calculating the charge density requires integration down to $x \rightarrow 0$. It is clear from the above that the LO chiral expansion and the limit $x \rightarrow 0$ do not commute. It explains why eq. (6.15) captures only the non-contact (intermediate $\pi N$ ) contribution to the charge density, and why the contact term has to be added separately. The LO chiral expansion correctly accounts for the total charge density; it is just not smooth enough at $y \rightarrow 0$ to allow for the charge to be distributed over partons with finite $x$ and recovered by integration over $x$. A resummation of chiral EFT at parametrically small $x$ in the logarithmic approximation has been proposed in refs. [36-38]; this approach "resolves" the delta function of the finite-order approximation into a finite-width function that allows integration over $x$. We emphasize that the small- $x$ problem discussed here is largely formal, and that chiral dynamics likely does not dominate the actual small- $x$ behavior of peripheral parton densities (for a discussion of chiral dynamics and resummation in the light of conventional small- $x$ physics, see ref. [22]).

\section{Summary and outlook}

Using the light-front representation of relativistic dynamics we have expressed the LO chiral EFT results for the nucleon's peripheral transverse densities in first-quantized form, as overlap integrals of the light-front wave functions of a chiral $\pi N$ system. The new 
representation is exactly equivalent to the second-quantized field-theoretical results and enables an intuitive understanding of chiral dynamics in close analogy to non-relativistic quantum mechanics. It reveals an inequality between the spin-independent and -dependent transverse densities, $\left|\widetilde{\rho}_{2}^{V}(b)\right|<\rho_{1}^{V}(b)$, which constrains the spatial distribution of charge and magnetization in the nucleon. It also offers a simple dynamical explanation why the inequality is almost saturated. The wave function representation permits straightforward numerical evaluation of the transverse densities and the underlying plus momentum distributions. It also connects the peripheral transverse charge density with the nucleon's peripheral partonic content (GPDs) generated by chiral dynamics.

Our studies reveal two interesting general aspects of chiral dynamics and peripheral nucleon structure. One is the essentially relativistic character of chiral dynamics in the parametric region of pion momenta $k=O\left(M_{\pi}\right)$. The large left-right asymmetry of the chiral densities in the transversely polarized nucleon is a genuine relativistic effect and results in a ratio very different from the non-relativistic estimate. It predicts the approximate equality $\left|\widetilde{\rho}_{2}^{V}(b)\right| \approx \rho_{1}^{V}(b)$ at $b=$ few $M_{\pi}^{-1}$, which can be tested experimentally. In this sense measurements of the nucleon's Dirac and Pauli form factors can directly attest to the relativistic nature of chiral dynamics in the nucleon's periphery (for a discussion of the prospects for extracting the peripheral transverse densities from nucleon form factor data, see refs. $[8,20])$.

The other interesting aspect is the role of the pion's orbital angular momentum in peripheral nucleon structure. It is seen most clearly in the case of transverse nucleon polarization, where a single pion orbital with $L=1$ accounts for both the spin-independent and -dependent densities and explains their properties. This places chiral dynamics in the context of contemporary studies of orbital angular momentum in relativistic systems and quantum field theory, inspired by the nucleon spin problem (for a recent review, see ref. [39]). The first-quantized light-front representation is an essential tool in defining the angular momentum content of relativistic systems and interpreting the dynamics, and is therefore natural for chiral dynamics.

The chiral two-pion exchange contribution studied in this work affects the isovector component of the nucleon charge densities. In the isoscalar component the chiral contribution starts with three-pion exchange and is strongly suppressed, because of its shorter range and its higher order in the chiral expansion. In this sense the isovector component computed here determines the large-distance behavior also of the individual proton and neutron densities, which are the sum of isovector and isoscalar components. The analysis of experimental data for the proton and neutron form factors at low $|t|$ should be done with dispersion-based parametrizations [32, 33], which have correct analytic properties (singularity structure) and smoothly combine the two-pion exchange contribution with the vector meson resonances determining the bulk of the transverse densities [31].

The methods developed in the present work can be applied to several related problems in nucleon structure. Of particular interest would be the study of chiral dynamics in the matrix elements of the energy-momentum tensor, whose transverse densities describe the spatial distributions of matter, momentum, and stress (or forces) in the nucleon. This would in particular allow one to confront the "particle-based" definition of orbital angular momentum in the light-front representation with the "field-theoretical" definition in terms 
of the energy-momentum tensor, and in this way test the various angular momentum sum rules proposed in the recent literature [40-44].

The light-front wave function representation of peripheral densities could also be extended to $\Delta$ isobar intermediate states. The $\Delta$ contribution to the transverse densities was computed in the dispersive approach in refs. $[8,20]$. While it is numerically small at distances $b=$ few $M_{\pi}^{-1}$ it plays an important role in ensuring the proper scaling behavior of the peripheral densities in the large- $N_{c}$ limit of QCD, where the $N-\Delta$ mass splitting scales as $M_{\Delta}-M_{N}=O\left(N_{c}^{-1}\right)$. The light-front wave functions for the $N \rightarrow \pi+\Delta$ transition can be defined in analogy to the $N \rightarrow \pi+N$ ones, cf. eq. (3.26). Light-front time-ordered calculations with higher-spin particles are generally plagued by ultraviolet divergences resulting from the breaking of rotational invariance. An advantage of the Lorentz-invariant approach taken in the present work (see section 3), in which the light-front representation is derived from the Feynman integrals, is that it maintains rotational invariance and avoids such divergences. It is thus particularly suited to including $\Delta$ intermediate states.

The nucleon's chiral component can be probed also in high-energy scattering processes in $\gamma N, e N, \pi N$, or $N N$ scattering (squared center-of-mass energies $s \gg 1 \mathrm{GeV}^{2}$ ), by selecting reaction channels and kinematic regions where the process happens predominantly on a pion at transverse distances $b=O\left(M_{\pi}^{-1}\right)$. Under certain conditions the amplitude for such processes can be expressed in terms of the light-front wave functions of the peripheral $\pi N$ system and the amplitude for the high-energy scattering process on the pion. These are the same light-front wave functions as those introduced in the present study of peripheral current matrix elements. Only the light-front formulation of chiral dynamics makes it possible to establish such a connection between low-energy and high-energy processes. This new connection greatly enlarges the number of experimental probes of chiral dynamics.

One example is hard exclusive electroproduction of mesons on a peripheral pion, $e+$ $N \rightarrow e^{\prime}+\pi+$ meson $+N^{\prime}$ at $Q^{2} \gg 1 \mathrm{GeV}^{2}$ and $x \ll M_{\pi} / M_{N}$, in the region where the invariant momentum transfer between the initial and final nucleons is $t_{N N^{\prime}} \equiv\left(p_{N}-p_{N}^{\prime}\right)^{2}=$ $O\left(M_{\pi}^{2}\right)$. Here the high-energy process on the pion, $e+\pi \rightarrow e^{\prime}+$ meson $+\pi$, probes the quark/gluon GPDs in the pion [21]. Such measurements could be performed at a future Electron-Ion Collider (EIC) with appropriate forward detectors for the pion and the recoiling nucleon. Another example is wide-angle quasi-elastic scattering on a peripheral pion, $\pi+N \rightarrow \pi+\pi+N^{\prime}$, in the region where $t_{N N^{\prime}}=O\left(M_{\pi}^{2}\right)$; here the high-energy process is described by the $\pi+\pi \rightarrow \pi+\pi$ elastic scattering amplitude at squared centerof-mass energies $s_{\pi \pi} \gg 1 \mathrm{GeV}^{2}$. An interesting consequence of chiral dynamics is that the cross sections for such "pion exchange" processes should have large transverse singlespin asymmetries, governed by the transverse spin dependence of the chiral $\pi N$ light-front wave function. This circumstance might further help to distinguish such processes from conventional "vacuum exchange" processes.

\section{A Light-front time-ordered formulation}

In section 3 the light-front wave function of the chiral $\pi N$ system is introduced as an element of a particular 3-dimensional reduction of the Feynman integrals for the nucleon form factor in relativistically invariant chiral EFT. In this appendix we show that this object 
is identical to the conventional wave function, defined as a transition matrix element in light-front time-ordered perturbation theory [11]. The correspondence is useful for relating our results to more phenomenological applications of light-front time-ordered perturbation theory, and for an eventual time-ordered formulation of chiral EFT.

In light-front quantization we consider the evolution of chiral effective field theory in light-front time $x^{+}=t+z$. It is governed by the Hamiltonian $H \equiv P^{-} / 2$, where $P^{-} \equiv P^{0}-P^{z}$ is the minus component of the energy-momentum 4 -vector of the field theory. In the interaction picture the Hamiltonian is split into a free part and an interaction, $H=H_{0}+H_{\text {int }}$. The eigenstates of the free Hamiltonian (which we denote as $|\ldots\rangle_{0}$ ) are nucleon and pion single-particle states, characterized by their light-front plus and transverse momenta, with eigenvalues given by the light-front energies,

$$
\begin{array}{rlrl}
H_{0}|N(p, \sigma)\rangle_{0} & =\frac{p^{-}}{2}|N(p, \sigma)\rangle_{0}, & & p^{-} \equiv \frac{\boldsymbol{p}_{T}^{2}+M_{N}^{2}}{2 p^{+}}, \\
H_{0}|\pi(k)\rangle_{0} & =\frac{k^{-}}{2}|\pi(k)\rangle_{0}, & k^{-} \equiv \frac{\boldsymbol{k}_{T}^{2}+M_{\pi}^{2}}{2 k^{+}},
\end{array}
$$

and are normalized as

$$
\begin{aligned}
{ }_{0}\left\langle N\left(p^{\prime}, \sigma^{\prime}\right) \mid N(p, \sigma)\right\rangle_{0} & =2 p^{+}(2 \pi)^{3} \delta\left(p^{+}-p^{+}\right) \delta^{(2)}\left(\boldsymbol{p}_{T}^{\prime}-\boldsymbol{p}_{T}\right) \delta\left(\sigma^{\prime}, \sigma\right), \\
{ }_{0}\left\langle\pi\left(k^{\prime}\right) \mid \pi(k)\right\rangle_{0} & =2 k^{+}(2 \pi)^{3} \delta\left(k^{\prime+}-k^{+}\right) \delta^{(2)}\left(\boldsymbol{k}_{T}^{\prime}-\boldsymbol{k}_{T}\right) .
\end{aligned}
$$

The interactions represent an operator in the product space spanned by the single-particle states and induce transitions between product states with different particle number. The evolution of a state from light-front time $x_{1}^{+}$to $x_{2}^{+}$is described by the action of the time evolution operator

$$
\begin{aligned}
S\left(x_{2}^{+}, x_{1}^{+}\right) & \equiv \mathrm{T} \exp \left[-i \int_{x_{1}^{+}}^{x_{2}^{+}} d x^{+} H_{\mathrm{int}}\left(x^{+}\right)\right], \\
H_{\text {int }}\left(x^{+}\right) & \equiv \exp \left(i H_{0} x^{+}\right) H_{\text {int }} \exp \left(-i H_{0} x^{+}\right),
\end{aligned}
$$

where the time ordering is in $x^{+}$and it is assumed that the original interaction operator $H_{\text {int }}$ does not explicitly depend on $x^{+}$.

Consider now the matrix element of the current operator at time $x^{+}=0$ between physical nucleon states with plus momenta $p_{1}^{+}=p_{2}^{+}=p^{+}$and transverse momenta such that $\boldsymbol{p}_{2 T}-\boldsymbol{p}_{1 T}=\boldsymbol{\Delta}_{T}$, cf. eq. (3.10). Assuming that the interaction is switched off adiabatically at $x^{+} \rightarrow \pm \infty$, the matrix element is given by

$$
\begin{aligned}
\left\langle N_{2}\left|J^{+}(0)\right| N_{1}\right\rangle & \equiv\left\langle N\left(p_{2}, \sigma_{2}\right)\left|J^{+}(0)\right| N\left(p_{1}, \sigma_{1}\right)\right\rangle \\
& ={ }_{0}\left\langle N\left(p_{2}, \sigma_{2}\right)\left|S(\infty, 0) J^{+}(0) S(0,-\infty)\right| N\left(p_{1}, \sigma_{1}\right)\right\rangle_{0} .
\end{aligned}
$$

A peripheral contribution originates from the components of the evolved nucleon state that contain a peripheral pion. In leading order of the interaction $H_{\text {int }}$ this component is a state with a nucleon and a single peripheral pion. The corresponding contribution to the matrix 
element is obtained by inserting free pion-nucleon intermediate states to the left and right of the current operator,

$$
\begin{aligned}
\left\langle N_{2}\left|J^{+}(0)\right| N_{1}\right\rangle= & \int \frac{d k_{1}^{+}}{(2 \pi) 2 k_{1}^{+}} \int \frac{d^{2} k_{1 T}}{(2 \pi)^{2}} \int \frac{d k_{2}^{+}}{(2 \pi) 2 k_{2}^{+}} \int \frac{d^{2} k_{2 T}}{(2 \pi)^{2}} \int \frac{d l^{+}}{(2 \pi) 2 l^{+}} \int \frac{d^{2} l_{T}}{(2 \pi)^{2}} \\
& \times_{0}\left\langle N\left(p_{2}, \sigma_{2}\right)|S(\infty, 0)| \pi\left(k_{2}\right) N(l, \sigma)\right\rangle_{0} \\
& \times_{0}\left\langle\pi\left(k_{2}\right)\left|J^{+}(0)\right| \pi\left(k_{1}\right)\right\rangle_{0} \\
& \times_{0}\left\langle\pi\left(k_{1}\right) N(l, \sigma)|S(0,-\infty)| N\left(p_{1}, \sigma_{1}\right)\right\rangle_{0} .
\end{aligned}
$$

The amplitude for the transition from the initial free nucleon state to the pion-nucleon intermediate state at time $x^{+}=0$ is

$$
\begin{aligned}
{ }_{0}\left\langle\pi\left(k_{1}\right) N(l, \sigma)|S(0,-\infty) \mathrm{LO}| N\left(p_{1}, \sigma_{1}\right)\right\rangle_{0} \\
={ }_{0}\left\langle\pi\left(k_{1}\right) N(l, \sigma)\left|(-i) \int_{-\infty}^{0} d x^{+} H_{\text {int }}\left(x^{+}\right)\right| N\left(p_{1}, \sigma_{1}\right)\right\rangle_{0} \\
={ }_{0}\left\langle\pi\left(k_{1}\right) N(l, \sigma)\left|H_{\text {int }}\right| N\left(p_{1}, \sigma_{1}\right)\right\rangle_{0}(-i) \int_{-\infty}^{0} d x^{+} e^{i\left(k_{1}^{-}+l^{-}-p_{1}^{-}-i 0\right) x^{+} / 2} \\
=-\frac{{ }_{0}\left\langle\pi\left(k_{1}\right) N(l, \sigma)\left|H_{\text {int }}\right| N\left(p_{1}, \sigma_{1}\right)\right\rangle_{0}}{\frac{1}{2}\left(k_{1}^{-}+l^{-}-p_{1}^{-}\right)} \\
\quad\left(p_{1}^{-}=\frac{\boldsymbol{p}_{1 T}^{2}+M_{N}^{2}}{p_{1}^{+}}, \quad k_{1}^{-}=\frac{\boldsymbol{k}_{1 T}^{2}+M_{\pi}^{2}}{k_{1}^{+}}, \quad l^{-}=\frac{\boldsymbol{l}_{T}^{2}+M_{N}^{2}}{l^{+}}\right),
\end{aligned}
$$

where the infinitesimal imaginary part of the light-front energies implies that the interaction is switched on adiabatically as $x^{+}$increases from $-\infty$. The interaction Hamiltonian is the integral of the interaction Hamiltonian density, which in turn is the negative of the interaction Lagrangian density,

$$
H_{\text {int }}=\frac{1}{2} \int d x^{-} d^{2} x_{T} \mathcal{H}_{\text {int }}(x)_{x^{+}=0}=-\frac{1}{2} \int d x^{-} d^{2} x_{T} \mathcal{L}_{\text {int }}(x)_{x^{+}=0} .
$$

We use translational invariance to evaluate the matrix element in eq. (A.9),

$$
\begin{aligned}
{ }_{0}\langle & \left.\pi\left(k_{1}\right) N(l, \sigma)\left|H_{\text {int }}\right| N\left(p_{1}, \sigma_{1}\right)\right\rangle_{0} \\
= & -\frac{1}{2} \int d x^{-} d^{2} x_{T}{ }_{0}\left\langle\pi\left(k_{1}\right) N(l, \sigma)\left|\mathcal{L}_{\text {int }}(x)\right| N\left(p_{1}, \sigma_{1}\right)\right\rangle_{0} \\
= & -\frac{1}{2} \int d x^{-} d^{2} x_{T} e^{i\left(k_{1}+l-p_{1}\right) x}{ }_{0}\left\langle\pi\left(k_{1}\right) N(l, \sigma)\left|\mathcal{L}_{\text {int }}(0)\right| N\left(p_{1}, \sigma_{1}\right)\right\rangle_{0} \\
= & -\left(2 \pi^{3}\right) \delta\left(k_{1}^{+}+l^{+}-p_{1}^{+}\right) \delta^{(2)}\left(\boldsymbol{k}_{1 T}+\boldsymbol{l}_{T}-\boldsymbol{p}_{1 T}\right) \\
& \times{ }_{0}\left\langle\pi\left(k_{1}\right) N(l, \sigma)\left|\mathcal{L}_{\text {int }}(0)\right| N\left(p_{1}, \sigma_{1}\right)\right\rangle_{0} .
\end{aligned}
$$

The LO transition matrix element eq. (A.9) can thus be represented as

$$
\begin{aligned}
{ }_{0}\left\langle\pi\left(k_{1}\right) N(l, \sigma)|S(0,-\infty) \mathrm{LO}| N\left(p_{1}, \sigma_{1}\right)\right\rangle_{0}= & 2 p_{1}^{+}\left(2 \pi^{3}\right) \delta\left(k_{1}^{+}+l^{+}-p_{1}^{+}\right) \\
& \times \delta^{(2)}\left(\boldsymbol{k}_{1 T}+\boldsymbol{l}_{T}-\boldsymbol{p}_{1 T}\right) \Psi \\
\Psi \equiv \Psi\left(y, \boldsymbol{k}_{T}, \boldsymbol{p}_{1 T} ; \sigma, \sigma_{1}\right) \equiv & \frac{{ }_{0}\left\langle\pi\left(k_{1}\right) N(l, \sigma)\left|\mathcal{L}_{\mathrm{int}}(0)\right| N\left(p_{1}, \sigma_{1}\right)\right\rangle_{0}}{p_{1}^{+}\left(k_{1}^{-}+l^{-}-p_{1}^{-}\right)},
\end{aligned}
$$


where $\Psi$ is the light-front wave function of the $\pi N$ component of the nucleon. It is invariant under longitudinal boosts and can be regarded as a function of the independent momentum variables $y=k_{1}^{+} / p_{1}^{+}, \boldsymbol{k}_{T}=\boldsymbol{k}_{1 T}-\boldsymbol{p}_{1 T}=-\boldsymbol{l}_{T}$, and $\boldsymbol{p}_{1 T}$. An analogous expression can be written for the complex conjugate matrix element describing the transition from the outgoing free pion-nucleon intermediate state to the final free nucleon state.

The wave function defined by eq. (A.13) is identical to the one introduced in the context of the reduction of the Feynman integral, eq. (3.26). Namely, the light-front energy denominator in eq. (A.13) is just the invariant mass difference eq. (3.17),

$$
p_{1}^{+}\left(k_{1}^{-}+l^{-}-p_{1}^{-}\right)=\frac{\boldsymbol{k}_{1 T}^{2}+M_{\pi}^{2}}{y}+\frac{\boldsymbol{l}_{T}^{2}+M_{N}^{2}}{1-y}-M_{N}^{2}-\boldsymbol{p}_{1 T}^{2}=\Delta \mathcal{M}^{2} .
$$

Furthermore, the transition matrix element of the Lagrangian density in the numerator in eq. (A.13) is just the $\mathrm{LO} \pi N$ vertex function eq. (3.23),

$$
\begin{aligned}
{ }_{0}\left\langle\pi\left(k_{1}\right) N(l, \sigma)\left|\mathcal{L}_{\text {int }}(0)\right| N\left(p_{1}, \sigma_{1}\right)\right\rangle_{0}= & \Gamma\left(y, \boldsymbol{k}_{T}, \boldsymbol{p}_{1 T} ; \sigma, \sigma_{1}\right) \\
& + \text { terms } \propto\left(k_{1}^{-}+l^{-}-p_{1}^{-}\right) .
\end{aligned}
$$

The expression quoted here is for the $p \rightarrow \pi^{0}+p$ isospin component of the matrix element; the other components follow from isospin invariance (cf. the comments after eq. (3.27)).

The final result for the leading-order peripheral contribution to the current matrix in the time-ordered approach is then obtained by (a) substituting the transition matrix elements in eq. (A.8) by the representation eq. (A.12), with the isospin factor $\sqrt{2}$ for the transitions $p \rightarrow \pi^{+}+n$ and $n \rightarrow \pi^{-}+p$; (b) substituting the explicit expression for the matrix element of the current operator between free (pointlike) charged pion states, ${ }_{0}\left\langle\pi^{( \pm)}\left(k_{2}\right)\left|J^{+}(0)\right| \pi^{( \pm)}\left(k_{1}\right)\right\rangle_{0}= \pm\left(k_{1}^{+}+k_{2}^{+}\right)$; (c) integrating over the redundant intermediate pion and nucleon momenta using the delta functions in eq. (A.12). The result for the isovector component is identical to eq. (3.27). Altogether this establishes the correspondence between the time-ordered and the invariant calculation of the LO chiral contribution to the peripheral densities.

Note that the transition matrix element eq. (A.15) calculated with the axial vector $\pi N N$ coupling (as appears in the original chiral Lagrangian) and the pseudoscalar coupling (as emerges from our reduction of the Feynman integral) differ by a term $\propto\left(k_{1}^{-}+l^{-}-p_{1}^{-}\right)$. This term cancels the energy denominator of the wave function and therefore results in a contact term in the matrix element, in agreement with the findings of section 3. One thus understands how the effective contact term eq. (3.9) appears in the time-ordered formulation. Calculation of the complete contact term contribution in the time-ordered formulation would be possible with a careful limiting procedure for the instantaneous exchanges. An advantage of our invariant formulation is that it allows us to calculate this contribution with minimum effort.

Notice: Authored by Jefferson Science Associates, LLC under U.S. DOE Contract No. DE-AC05-06OR23177. The U.S. Government retains a non-exclusive, paid-up, irrevocable, world-wide license to publish or reproduce this manuscript for U.S. Government purposes. 
Open Access. This article is distributed under the terms of the Creative Commons Attribution License (CC-BY 4.0), which permits any use, distribution and reproduction in any medium, provided the original author(s) and source are credited.

\section{References}

[1] D.E. Soper, The Parton Model and the Bethe-Salpeter Wave Function, Phys. Rev. D 15 (1977) 1141 [nSPIRE].

[2] M. Burkardt, Impact parameter dependent parton distributions and off forward parton distributions for $\zeta \rightarrow$ 0, Phys. Rev. D 62 (2000) 071503 [Erratum ibid. D 66 (2002) 119903] [hep-ph/0005108] [INSPIRE].

[3] G.A. Miller, Charge Density of the Neutron, Phys. Rev. Lett. 99 (2007) 112001 [arXiv: 0705.2409] [INSPIRE].

[4] G.A. Miller, Transverse Charge Densities, Ann. Rev. Nucl. Part. Sci. 60 (2010) 1 [arXiv: 1002.0355] [INSPIRE].

[5] M. Burkardt, Impact parameter space interpretation for generalized parton distributions, Int. J. Mod. Phys. A 18 (2003) 173 [hep-ph/0207047] [INSPIRE].

[6] C.E. Carlson and M. Vanderhaeghen, Empirical transverse charge densities in the nucleon and the nucleon-to-Delta transition, Phys. Rev. Lett. 100 (2008) 032004 [arXiv:0710.0835] [INSPIRE].

[7] S. Venkat, J. Arrington, G.A. Miller and X. Zhan, Realistic Transverse Images of the Proton Charge and Magnetic Densities, Phys. Rev. C 83 (2011) 015203 [arXiv:1010.3629] [INSPIRE].

[8] C. Granados and C. Weiss, Chiral dynamics and peripheral transverse densities, JHEP 01 (2014) 092 [arXiv: 1308.1634] [INSPIRE].

[9] P.A.M. Dirac, Forms of Relativistic Dynamics, Rev. Mod. Phys. 21 (1949) 392 [InSPIRE].

[10] H. Leutwyler and J. Stern, Relativistic Dynamics on a Null Plane, Annals Phys. 112 (1978) 94 [INSPIRE].

[11] S.J. Brodsky, H.-C. Pauli and S.S. Pinsky, Quantum chromodynamics and other field theories on the light cone, Phys. Rept. 301 (1998) 299 [hep-ph/9705477] [INSPIRE].

[12] C. Granados and C. Weiss, Quantum-mechanical picture of peripheral chiral dynamics, arXiv:1503.02055 [INSPIRE].

[13] M.G. Schmidt, A simple connection between covariant Feynman formalism and time ordered perturbation theory in the infinite momentum frame, Phys. Rev. D 9 (1974) 408 [InSPIRE].

[14] A. Fernandez-Pacheco, J.A. Grifols and I.A. Schmidt, A Relativistic Description of the Electromagnetic Form-factor of the Deuteron, Nucl. Phys. B 151 (1979) 518 [InSPIRE].

[15] M. Sawicki, Soft charge form-factor of the pion, Phys. Rev. D 46 (1992) 474 [INSPIRE].

[16] W. Jaus, Covariant analysis of the light front quark model, Phys. Rev. D 60 (1999) 054026 [INSPIRE].

[17] C.-R. Ji, W. Melnitchouk and A.W. Thomas, Equivalence of pion loops in equal-time and light-front dynamics, Phys. Rev. D 80 (2009) 054018 [arXiv:0906.3497] [INSPIRE]. 
[18] M. Burkardt, K.S. Hendricks, C.-R. Ji, W. Melnitchouk and A.W. Thomas, Pion momentum distributions in the nucleon in chiral effective theory, Phys. Rev. D 87 (2013) 056009 [arXiv: 1211.5853] [INSPIRE].

[19] C.-R. Ji, W. Melnitchouk and A.W. Thomas, Anatomy of relativistic pion loop corrections to the electromagnetic nucleon coupling, Phys. Rev. D 88 (2013) 076005 [arXiv:1306.6073] [INSPIRE].

[20] M. Strikman and C. Weiss, Quantifying the nucleon's pion cloud with transverse charge densities, Phys. Rev. C 82 (2010) 042201 [arXiv: 1004.3535] [INSPIRE].

[21] M. Strikman and C. Weiss, Chiral dynamics and the growth of the nucleon's gluonic transverse size at small x, Phys. Rev. D 69 (2004) 054012 [hep-ph/0308191] [INSPIRE].

[22] M. Strikman and C. Weiss, Chiral dynamics and partonic structure at large transverse distances, Phys. Rev. D 80 (2009) 114029 [arXiv:0906.3267] [InSPIRE].

[23] T. Becher and H. Leutwyler, Baryon chiral perturbation theory in manifestly Lorentz invariant form, Eur. Phys. J. C 9 (1999) 643 [hep-ph/9901384] [INSPIRE].

[24] J. Gasser, M.E. Sainio and A. Svarc, Nucleons with Chiral Loops, Nucl. Phys. B 307 (1988) 779 [inSPIRE].

[25] V. Bernard, N. Kaiser, J. Kambor and U.G. Meissner, Chiral structure of the nucleon, Nucl. Phys. B 388 (1992) 315 [InSPIRE].

[26] B. Kubis and U.-G. Meissner, Low-energy analysis of the nucleon electromagnetic form-factors, Nucl. Phys. A 679 (2001) 698 [hep-ph/0007056] [INSPIRE].

[27] N. Kaiser, Spectral functions of isoscalar scalar and isovector electromagnetic form-factors of the nucleon at two loop order, Phys. Rev. C 68 (2003) 025202 [nucl-th/0302072] [INSPIRE].

[28] L.L. Frankfurt and M.I. Strikman, High-Energy Phenomena, Short Range Nuclear Structure and QCD, Phys. Rept. 76 (1981) 215 [INSPIRE].

[29] S.L. Adler, Sum rules for the axial vector coupling constant renormalization in beta decay, Phys. Rev. 140 (1965) B736 [Erratum ibid. 149 (1966) 1294] [Erratum ibid. 175 (1968) 2224] [INSPIRE].

[30] W.I. Weisberger, Unsubtracted Dispersion Relations and the Renormalization of the Weak Axial Vector Coupling Constants, Phys. Rev. 143 (1966) 1302 [INSPIRE].

[31] G.A. Miller, M. Strikman and C. Weiss, Realizing vector meson dominance with transverse charge densities, Phys. Rev. C 84 (2011) 045205 [arXiv: 1105.6364] [InSPIRE].

[32] M.A. Belushkin, H.W. Hammer and U.G. Meissner, Dispersion analysis of the nucleon form-factors including meson continua, Phys. Rev. C 75 (2007) 035202 [hep-ph/0608337] [INSPIRE].

[33] I.T. Lorenz, H.W. Hammer and U.-G. Meissner, The size of the proton - closing in on the radius puzzle, Eur. Phys. J. A 48 (2012) 151 [arXiv:1205.6628] [INSPIRE].

[34] G. Hohler et al., Analysis of Electromagnetic Nucleon Form-Factors, Nucl. Phys. B 114 (1976) 505 [inSPIRE].

[35] X.-D. Ji, Deeply virtual Compton scattering, Phys. Rev. D 55 (1997) 7114 [hep-ph/9609381] [INSPIRE].

[36] N. Kivel and M.V. Polyakov, Breakdown of chiral expansion for parton distributions, Phys. Lett. B 664 (2008) 64 [arXiv:0707.2208] [INSPIRE]. 
[37] N. Kivel, M.V. Polyakov and A. Vladimirov, Large-N Summation of Chiral Logs for Generalized Parton Distributions, Phys. Rev. D 79 (2009) 014028 [arXiv:0809.2064] [INSPIRE].

[38] I.A. Perevalova, M.V. Polyakov, A.N. Vall and A.A. Vladimirov, Chiral Inflation of the Pion Radius, arXiv:1105.4990 [INSPIRE].

[39] E. Leader and C. Lorcé, The angular momentum controversy: What's it all about and does it matter?, Phys. Rept. 541 (2014) 163 [arXiv: 1309.4235] [INSPIRE].

[40] X.-D. Ji, Gauge-Invariant Decomposition of Nucleon Spin, Phys. Rev. Lett. 78 (1997) 610 [hep-ph/9603249] [INSPIRE].

[41] X. Ji, X. Xiong and F. Yuan, Proton Spin Structure from Measurable Parton Distributions, Phys. Rev. Lett. 109 (2012) 152005 [arXiv:1202.2843] [INSPIRE].

[42] X. Ji, X. Xiong and F. Yuan, Probing Parton Orbital Angular Momentum in Longitudinally Polarized Nucleon, Phys. Rev. D 88 (2013) 014041 [arXiv:1207.5221] [INSPIRE].

[43] X. Ji, X. Xiong and F. Yuan, Transverse Polarization of the Nucleon in Parton Picture, Phys. Lett. B 717 (2012) 214 [arXiv:1209.3246] [INSPIRE].

[44] E. Leader, New relation between transverse angular momentum and generalized parton distributions, Phys. Rev. D 85 (2012) 051501 [arXiv:1109.1230] [INSPIRE]. 